\title{
LA VEGETACIÓN DEL NORTE DEL PERÚ: DE LOS BOSQUES A LA JALCA EN CAJAMARCA
}

\author{
Antonio GALÁN DE MERA ${ }^{1 *}$, Isidoro SÁNCHEZ VEGA $\dagger^{2}$, Juan MONTOYA QUINO², \\ Eliana LINARES PEREA ${ }^{3}$, José CAMPOS DE LA CRUZ ${ }^{4}$ \\ y José Alfredo VICENTE ORELLANA ${ }^{1}$ \\ ${ }^{1}$ Laboratorio de Botánica, Facultad de Farmacia, Universidad San Pablo-CEU, Apartado 67, 28660 \\ Boadilla del Monte, Madrid, España \\ ${ }^{2}$ Laboratorio de Botánica, Facultad de Ciencias de la Salud (Farmacia), Universidad Privada Antonio \\ Guillermo Urrelo (UPAGU), Jr. José Sabogal 913, Cajamarca, Perú \\ ${ }^{3}$ Estudios Fitogeográficos del Perú, Sánchez Cerro 219, Manuel Prado, Paucarpata, Arequipa, Perú \\ ${ }^{4}$ Museo de Historia Natural, Universidad Nacional Mayor de San Marcos, \\ Apartado 140434, Lima 14, Perú \\ * Autor para correspondencia: agalmer@ceu.es
}

Recibido el 22 de septiembre de 2015, aceptado para su publicación el 27 de octubre de 2015

RESUMEN. La vegetación del Norte del Perú: De los bosques a la jalca en Cajamarca. El departamento de Cajamarca, en el Norte del Perú, se caracteriza en general por un clima más húmedo que el resto del territorio andino peruano occidental. Teniendo en cuenta el paralelismo entre el clima y las unidades de vegetación que ofrecen los pisos bioclimáticos, los ombrotipos, la geología y los suelos, y levantando inventarios según la metodología de Braun-Blanquet, estudiamos la vegetación de este territorio. Se describen 2 comunidades vegetales, 8 asociaciones, 3 subasociaciones, 3 alianzas, 1 orden y 1 clase fitosociológica. Como resultado, se analizan la comunidad de Cecropia montana y Heliocarpus americanus (termotropical subhúmedo-húmeda) y de Muntingia calabura y Hura crepitans (infratropical seco-subhúmeda), ambas de la clase Nectandro laevis-Licarietea canellae presente en el norte del territorio. Hacia el sur, los bosques lauroides y esclerófilos están representados por las nuevas asociaciones Axinaeo nitidae-Podocarpetum oleifolii (bosques mesotropicales, húmedo-hiperhúmedos), Verbesino auriculigerae-Siparunetum muricatae (bosques mesotropicales húmedo-hiperhúmedos de áreas alteradas), Berberido beauverdianae-Myrcianthetum myrsinoidis (bosques del piso mesotropical inferior seco-subhúmedo) y Aristeguietio discolorisKageneckietum lanceolatae (bosques del piso mesotropical superior seco-subhúmedo), todas agrupadas en la nueva alianza Monnino pilosae-Myrcianthion myrsinoidis (Pruno rigidae-Oreopanacetea floribundi). Las asociaciones nuevas Baccharito latifoliae-Monactinetum flaverioidis (supra-mesotropical subhúmedo-seca) y Monactino flaverioidis-Colignonietum parviflorae (mesotropical húmeda) son las arbustedas sucesionales de los bosques y se agrupan en la alianza Otholobio munyensis-Rubion robusti all. nova y en la nueva clase andina Clematido peruvianae-Baccharitetea latifoliae. Se describen dos nuevas asociaciones de los pajonales del páramo ("jalca”) con sus subasociaciones: Calamagrostio tarmensis-Hypericetum laricifolii (pajonales supratropicales húmedos del sector Chota-Contumazá), con la subasociación cortaderietosum nitidae en áreas rocosas, y Agrostio tolucensis-Paspaletum bonplandiani (pajonales orotropicales húmedoshiperhúmedos del sector Chota-Contumazá), con la subasociación puyetosum fastuosae en suelos húmedos, y calamagrostietosum curvulae como una subasociación de transición hacia los pajonales de la puna del centro del Perú. Además, hemos reconocido y lectotipificado la asociación supratropical húmeda Oreobolo goeppingieri-Hypericetum laricifolii, característica del sector biogeográfico Loja-Cutervo. Todas estas asociaciones de pastizales pertenecen a la nueva alianza Agrostio tolucensis-Paspalion bonplandiani y al nuevo orden Agrostio tolucensis-Paspaletalia bonplandiani (Calamagrostietea vicunarum). Por otra parte, presentamos un esquema sintaxonómico con todas las clases fitosociológicas estudiadas por el 
momento en el departamento de Cajamarca, incluyendo bosques secos y vegetación con cactus y otras plantas suculentas (Acacio macracanthae-Prosopidetea pallidae), alisedas (Alnetea acuminatae), bosques altoandinos (Polylepidetea tarapacano-besseri), y vegetación helofítica de riveras (Tessario integrifoliaeBaccharitetea salicifoliae). Como resultado de este estudio fitosociológico comentamos la biogeografía del $\mathrm{N}$ del Perú estableciendo los sectores Loja-Cutervo y Chota-Contumazá, y finalmente hemos cartografiado la vegetación potencial de Cajamarca.

Palabras clave. Vegetación, fitosociología, páramo, jalca, Andes, Norte de Perú, Cajamarca.

ABSTRACT. Vegetation of Northern Peru: From the humid forests to the "jalca" in Cajamarca. The Department of Cajamarca, in Northern Peru, is generally characterized by a more general humid climate that in the rest of the occidental Andean Peruvian territory. Taking account of the parallelism between climate and vegetation units offered by bioclimatic belts and ombrotypes, geology and soils, and making plots according to the Braun-Blanquet methodology, the vegetation of the territory was studied. Two plant communities, 8 associations, 3 subassociations, 3 alliances, 1 order and 1 phytosociological class are described. As a result, we have analyzed the Cecropia montana and Heliocarpus americanus (thermotropical subhumid-humid) and the Muntingia calabura and Hura crepitans (infratropical dry-subhumid) plant communities, both belonging to the Nectandro laevis-Licarietea canellae class in the North of the territory. To the South, laurel like and sclerophyllous forests are represented by the new associations Axinaeo nitidae-Podocarpetum oleifolii (mesotropical, humid-hyperhumid forests), Verbesino auriculigerae-Siparunetum muricatae (mesotropical humid-hyperhumid forests on disturbed areas), Berberido beauverdianae-Myrcianthetum myrsinoidis (lower mesotropical dry-subhumid forests), and Aristeguietio discoloris-Kageneckietum lanceolatae (upper mesotropical dry-subhumid forests), all grouped into the new alliance Monnino pilosae-Myrcianthion myrsinoidis (Pruno rigidae-Oreopanacetea floribundi). The supra-mesotropical, subhumid-dry Baccharito latifoliae-Monactinetum flaverioidis and the mesotropical humid Monactino flaverioidis-Colignonietum parviflorae new associations are the successional shrublands of the forests, grouped into the new alliance Otholobio munyensis-Rubion robusti and into the new Andean class Clematido peruvianae-Baccharitetea latifoliae. Two new associations for the paramo ("jalca") grasslands have been described with their subassociations: Calamagrostio tarmensis-Hypericetum laricifolii (supratropical humid grasslands from the Chota-Contumazá biogeographical sector), with cortaderietosum nitidae subassociation on rocky areas, and Agrostio tolucensis-Paspaletum bonplandiani (orotropical humid-hyperhumid grasslands from the Chota-Contumazá biogeographical sector), with puyetosum fastuosae subassociation on humid soils, and calamagrostietosum curvulae as transitional subassociation to the puna grasslands in Central Peru. Furthermore, we have recognized and lectotypified the supratropical humid association Oreobolo goeppingieriHypericetum laricifolii from the Loja-Cutervo biogeographical sector. These grassland associations belong to the new alliance Agrostio tolucensis-Paspalion bonplandiani and to the new order Agrostio tolucensisPaspaletalia bonplandiani (Calamagrostietea vicunarum class). Besides, we present a syntaxonomical scheme with all the phytosociological class studied for the moment in the Department of Cajamarca, including dry forest and succulent vegetation with cactus (Acacio macracanthae-Prosopidetea pallidae), Andean alder forests (Alnetea acuminatae), high Andean forests (Polylepidetea tarapacano-besseri), and helophytic river vegetation (Tessario integrifoliae-Baccharitetea salicifoliae).

As a result of the study of the phytosociological units of Cajamarca, we comment the biogeography of Northern Peru establishing the Loja-Cutervo and Chota-Contumazá sectors, and finally, we have maped the potential vegetation of Cajamarca.

Key words. Vegetation, phytosociology, páramo, jalca, Andes, Northern Peru, Cajamarca.

\section{INTRODUCCIÓN}

El Norte del Perú comprende los territorios situados al $\mathrm{N}$ del paralelo $8^{\circ} \mathrm{S}$ hasta la frontera con Ecuador (Mostacero León et al., 1996). En este espacio se observa un cambio de la orografía, clima y vegetación respecto al resto del país. La depresión de Huancabamba $\left(5^{\circ} 45^{\text {, }}\right.$ 
a $6^{\circ} 19^{\prime}$ S), que incluye el punto más bajo de los Andes occidentales (2145 m), y las pequeñas alineaciones montañosas transversales son los hitos orográficos más importantes, pues originan el aislamiento necesario para la presencia de un elevado número de endemismos (Weigend, 2002). La climatología está marcada al occidente por la desaparición de la influencia de la corriente fría marítima de Humboldt y el efecto de las lluvias provocadas por la corriente cálida ecuatorial, y al oriente por las procedentes de la Amazonía (Galán de Mera \& Vicente Orellana, 2007). Estas lluvias aportan humedad a ambas vertientes de los Andes, origen de bosques diversos, vicariantes de los de Colombia (Rangel, 2015) y Ecuador (Bussmann, 2003; Izco, 2013) y el establecimiento de páramo o jalca en las cumbres.

Son ya numerosos los trabajos sobre la flora de los bosques del Norte del Perú (Sagástegui Alva, 1989; Sagástegui Alva et al., 1995; Juárez et al., 2005; Llatas Quirós \& López-Mesones, 2005; Weigend et al., 2005; Weigend \& Rodríguez, 2005) y el páramo (Mostacero León et al., 1996; Luteyn, 1999; Marcelo Peña et al., 2006; Sánchez Vega \& Sánchez Rojas, 2012), aunque como indican Jørgensen et al. (1995) existen muy pocas comparaciones sobre la composición florística de los territorios adyacentes entre Ecuador y Perú. Los trabajos fitosociológicos en el $\mathrm{N}$ del Perú son bastante escasos, con excepción de la aproximación inicial de Galán de Mera et al. (2013), las aportaciones de Sabogal (2014) y Peyre (2015), y el ensayo sobre los bosques secos y vegetación con plantas suculentas de Galán de Mera et al. (2015).

Como continuación de los estudios fitosociológicos realizados previamente en el sur (Galán de Mera \& Linares Perea, 2012) y en el centro de los Andes peruanos (Galán de Mera et al., 2004, 2014), con este trabajo seguimos abordando las comunidades vegetales que se desarrollan por encima del paralelo $8^{\circ} \mathrm{S}$. Con ello, además, aportamos algunas novedades sobre la biogeografía del Norte del Perú.

\section{MATERIAL Y MÉTODOS}

\section{El territorio estudiado}

En estas primeras descripciones de unidades fitosociológicas del $\mathrm{N}$ del Perú nos centraremos en el departamento de Cajamarca por representar un espacio bien diferenciado respecto a los Andes comprendidos entre Ancash (centro de Perú) y Tacna (límite sur de Perú).

El departamento de Cajamarca está situado entre la latitud $7^{\circ} 32$ 'S y la frontera con Ecuador (458’S). Al E limita con el departamento de Amazonas a través del río Marañón hasta su confluencia con el Chinchipe; al O encontramos los valles áridos que se continúan en los departamentos de Lambayeque y Piura, y al O y S en el departamento de La Libertad. Excepto el río Marañón, que recorre de $\mathrm{S}$ a $\mathrm{N}$ gran parte de la frontera oriental del territorio, todos los demás ríos suelen tener un trazado transversal separando relieves que en pocos casos superan los $4000 \mathrm{~m}$ (El Chunque- $4061 \mathrm{~m}$, Cerro Pedregal- 4018 m, Cerro Azul- 4183 m, Chonta Pajcha- 4006 m y San Antonio- 4496 m). Las mayores altitudes de la región se encuentran concentradas en el S, lo que indica una disminución progresiva de las altitudes hasta la depresión de Huancabamba, donde finalizan hacia el N los Andes Centrales (Sánchez Vega \& Sánchez Rojas, 2012).

La geología del territorio está ligada al mar interior paleozoico de América del Sur y al vulcanismo terciario de tipo continental que configuró la mayor parte del relieve andino (Lisson, 1980). Los materiales más antiguos se encuentran al $\mathrm{N}$ con el complejo metamórfico precámbrico del Marañón, y al E con los afloramientos cretácicos del Marañón, que se extienden hacia el interior, como en los alrededores de Cajamarca y Chetilla. El resto 
son materiales volcánicos terciarios más o menos antiguos, a veces salpicados por rocas intrusivas, sobre todo granitos y granodioritas (Instituto de Geología y Minería, 1975). La menor altitud de los Andes en esta latitud parece debida a la disminución de la influencia de la placa de Nazca y a hundimientos ocurridos desde el Paleozoico (Raynel et al., 2013).

En el O de América del Sur, durante el Mioceno, la combinación entre la situación del anticiclón del Pacífico Sur, debida a la corriente de Humboldt, y la sombra de lluvia de los Andes diferenciaron elementos florísticos pre-adaptados a la aridez de las vertientes occidentales andinas (Galán de Mera et al., 2012). Sin embargo, la corriente de Humboldt deja de influir sobre la vegetación hacia el paralelo $8^{\circ} \mathrm{S}$, permitiendo la llegada de masas de aire cálido a las laderas occidentales, que originan precipitaciones que van en aumento desde el departamento de La Libertad hacia el N. El departamento de Cajamarca presenta bosques húmedos al $\mathrm{E}$ y $\mathrm{O}$, con páramo sobre los $3000 \mathrm{~m}$.

\section{Bioclimatología, datos meteorológicos, nomenclatura y colecciones}

Para establecer un paralelismo entre el clima y la vegetación hemos utilizado la metodología bioclimática de Rivas-Martínez (Rivas-Martínez \& Rivas-Sáenz, 1996-2015), con la que podemos establecer cinturones altitudinales y latitudinales de vegetación (pisos bioclimáticos) basadas en el índice de termicidad $[\mathrm{It}=\mathrm{T}+\mathrm{M}+\mathrm{m}) 10 ; \mathrm{T}$ : temperatura media anual $\left({ }^{\circ} \mathrm{C}\right), \mathrm{M}$ : temperatura media de las máximas del mes más frío $\left({ }^{\circ} \mathrm{C}\right), \mathrm{m}$ : temperatura media de las mínimas del mes más frío $\left.\left({ }^{\circ} \mathrm{C}\right)\right]$. Para valorar la disponibilidad del agua de las precipitaciones por las plantas, este mismo autor estableció el índice ombrotérmico anual $[\mathrm{Io}=(\mathrm{Pp} / \mathrm{Tp}) 10$; $\mathrm{Pp}$ : precipitación positiva anual en mm (suma de las precipitaciones de los meses en que $\mathrm{T}>$ $0^{\circ} \mathrm{C}$ ), Tp: sumatorio en decímas de ${ }^{\circ} \mathrm{C}$ de las temperaturas medias de los meses $\left.>0^{\circ} \mathrm{C}\right]$. Los intervalos de Io constituyen los ombrotipos. En el territorio estudiado los pisos bioclimáticos son: infratropical (horizonte superior, It = 710-800), termotropical (horizonte inferior, It =600-710; horizonte superior, It $=490-600$ ), mesotropical (horizonte inferior, It $=405-490$; horizonte superior, $\mathrm{It}=320-405$ ), supratropical (horizonte inferior, It $=240-320$; horizonte superior, It $=160-240$ ) y orotropical (horizonte inferior, $\mathrm{It}=120-160)$. Los ombrotipos son los siguientes: semiárido (horizonte inferior, Io $=$ 1.0-1.5; horizonte superior, Io $=1.5-2.0$ ), seco (horizonte inferior, Io $=2.0-2.8$; horizonte superior, Io $=2.8-3.6$ ), subhúmedo (horizonte inferior, Io $=3.6-4.8$; horizonte superior, Io = 4.8-6.0), húmedo (horizonte inferior, Io = 6.0-9.0; horizonte superior, Io $=9.0-12.0$ ) e hiperhúmedo (horizonte inferior, Io = 12.0-18.0) (tab. 1).

Los datos meteorológicos para la determinación de los pisos bioclimáticos y ombroclimas se obtuvieron del Servicio Nacional de Meteorología e Hidrología del Perú (SENAMHI) y de la base de datos Global Bioclimatics (Rivas-Martínez \& Rivas-Sáenz, 1996-2015).

La nomenclatura y autorías de las plantas citadas siguen a The Plant List (2015). Para su determinación se han consultado los herbarios CPUN, F, MO y USM, conservándose el material utilizado en CPUN con duplicados en USM. Además se han manejado las principales guías territoriales sobre la flora (Gentry, 1993; Sagástegui Alva et al., 1999, 2003).

La sintaxonomía está basada en el Código Internacional de Nomenclatura Fitosociológica (Weber et al., 2000).

\section{La vegetación}

El estudio de la vegetación está basado en inventarios fitosociológicos (Braun-Blanquet, 1979) levantados en áreas de unos $100 \mathrm{~m}^{2}$ para los pastizales y arbustedas, y $500 \mathrm{~m}^{2}$ para los bosques, de acuerdo con el principio del área mínima (Dengler et al., 2008). Estos inventarios 


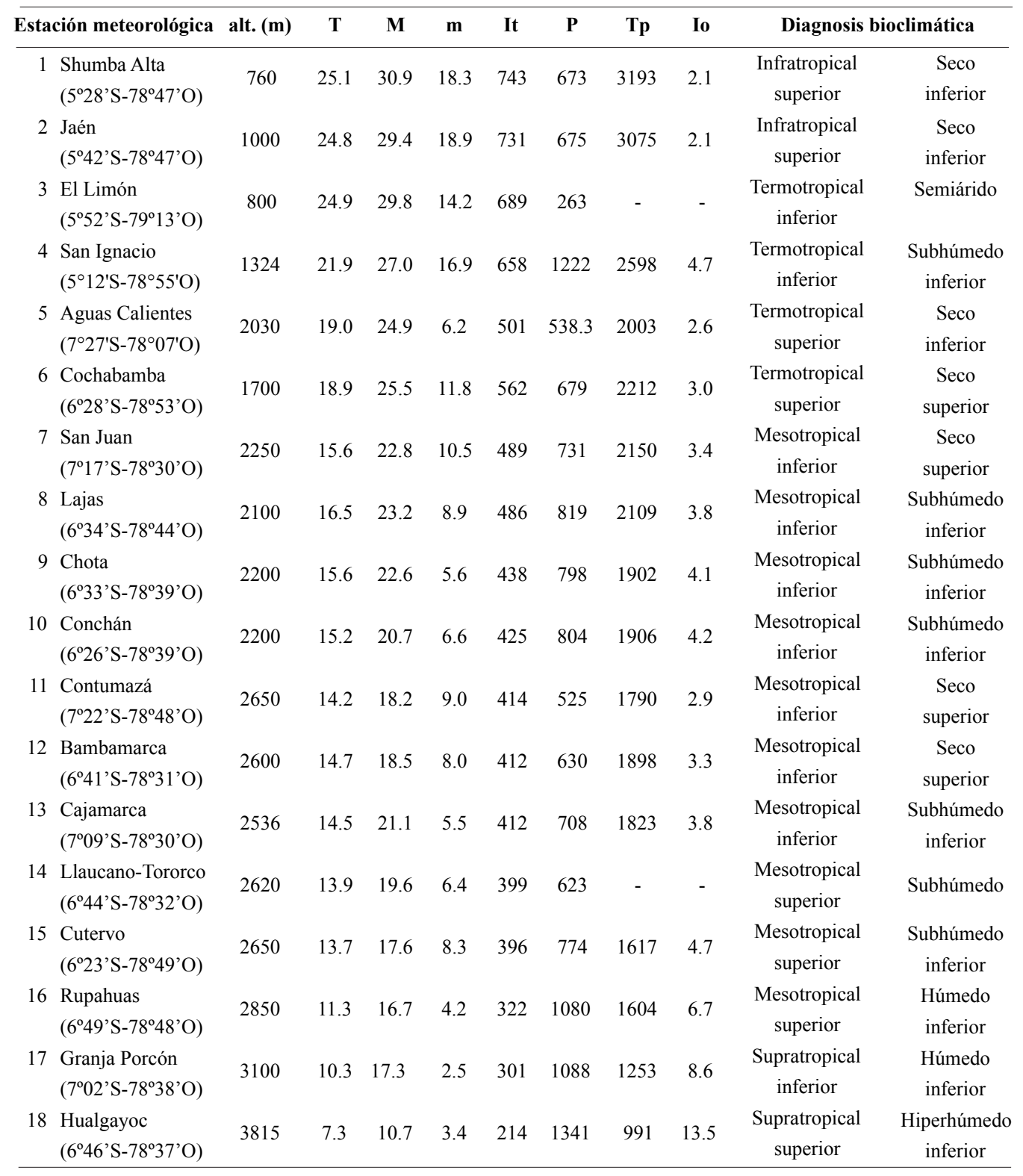

Tabla 1. Variables climáticas, índices bioclimáticos y diagnosis bioclimática de las estaciones meteorológicas del territorio estudiado, de acuerdo con la metodología de Rivas-Martínez (Rivas-Martínez \& Rivas-Sáenz, 1996-2015)[T: temperatura media anual $\left({ }^{\circ} \mathrm{C}\right), \mathrm{M}$ : temperatura media de las máximas del mes más frío $\left({ }^{\circ} \mathrm{C}\right)$, $\mathrm{m}$ : temperatura media de las mínimas del mes más frío $\left({ }^{\circ} \mathrm{C}\right)$, It: índice de termicidad, P: precipitación anual $(\mathrm{mm})$, Tp: temperatura positiva anual, Io: índice ombrotérmico anual]. Climatic variables, bioclimatic indexes and bioclimatic diagnosis of the meteorological stations of the studied territory according to the methodology of Rivas-Martinez (Rivas-Martínez \& Rivas-Sáenz, 1996-2015) [T: average annual temperature $\left({ }^{\circ} \mathrm{C}\right)$, M: average highest temperature in the coldest month $\left({ }^{\circ} \mathrm{C}\right), \mathrm{m}$ : average lowest temperature in the coldest month $\left({ }^{\circ} \mathrm{C}\right)$, It: thermicity index, $P$ : annual precipitation $(\mathrm{mm})$, Tp: yearly positive temperature; Io:yearly ombrothermic index]. 
han sido dispuestos en tablas fitosociológicas teniendo en cuenta los distintos estratos de la vegetación y la fidelidad que guardan las plantas entre sí (Géhu \& Rivas-Martínez, 1981). Para la elección de las plantas características de las unidades fitosociológicas hemos estudiado su distribución altitudinal y latitudinal con la base de datos Tropicos (2015), algunos catálogos florísticos (Brako \& Zarucchi, 1993; Jørgensen \& León-Yáñez, 1999; Luteyn, 1999), y otros trabajos sobre vegetación (Lauer et al., 2001; Gentry, 1995-2015; Phillips \& Miller, 2002; Bussmann, 2003; Galán de Mera et al., 2004, 2014; Galán de Mera, 2005; Izco, 2013; Sabogal, 2014) donde se emplearon índices de abundancia.

Con la disposición de las asociaciones en cliseries altitudinales (Rivas-Martínez \& Rivas-Sáenz, 1996-2015), estudiando su posición y contactos fitotopográficos, se discute la biogeografía del $\mathrm{N}$ del Perú en relación con los territorios adyacentes.

\section{RESULTADOS Y DISCUSIÓN}

\section{Novedades fitosociológicas}

1. Las pluvisilvas andinas del $\mathrm{N}$ del departamento de Cajamarca (Clase Nectandro laevis-Licarietea canellae Izco 2013)

Comunidad de Cecropia montana Warb. ex Snethl. y Heliocarpus americanus L. [Tabla 2]

Bosques termotropicales, subhúmedohúmedos cuyo dosel alcanza los $30 \mathrm{~m}$ de altura y se asientan, a veces alcanzando una pendiente del $60 \%$, sobre suelos profundos derivados de materiales graníticos. En el departamento de Cajamarca se extienden por todo el N, abarcando la mayor parte de la provincia de San Ignacio sobre los $1500 \mathrm{~m}$ hasta las alineaciones montañosas $(\sim 2500$ $\mathrm{m})$ que se extienden orientadas al NE-SO entre el cerro Pergacucho y Cutervo (Gentry, 1995-2015; Phillips \& Miller, 2002). Se trata de una continuación de los bosques húmedos ecuatorianos (Bussmann, 2003; Izco, 2013) que incluso alcanzan más al S el departamento de San Martín (Schjellerup et al., 2003), como indican las plantas características de la alianza Tibouchino lepidotae-Vismion tomentosae Izco 2013 (= Cecropio montanae-Isertion laevis Bussmann 2002): Cecropia montana, Cinchona pubescens Vahl, Isertia laevis (Triana) Boom, Myrsine latifolia (Ruiz \& Pav.) Spreng. y Weinmannia spruceana Engl. Por supuesto, hemos encontrado características del orden Nectandro laevis-Clusietalia emarginatae Izco 2013 (= Alzateetalia verticillatae Bussmann 2002) y de la clase Nectandro laurel-Licarietea canellae Izco 2013, todo ello acompañado de un estrato nemoral de helechos (Elaphoglossum cuspidatum (Willd.) T. Moore, Nephrolepis cordifolia (L.) C. Presl, Serpocaulon caceresii (Sodiro) A.R. Sm. y Thelypteris leprieurii (Hook.) R.M. Tryon) y aráceas (Anthurium giganteum Engl. y A. trilobum Lindl.).

Los bosques de Cecropia montana y Heliocarpus americanus se encuentran muy alterados por los cultivos de café, dando lugar a espacios donde Pennisetum peruvianum Trin. y Pteridium arachnoideum (Kaulf.) Maxon se hacen constantes.

Comunidad de Muntingia calabura L. y Hura crepitans L. [Tabla 2]

Bosque infratropical seco-subhúmedo que se sitúa catenalmente por debajo de la comunidad anterior, entre 500 y $1500 \mathrm{~m}$, ocupando las partes bajas de las cuencas del río Tabaconas y sus afluentes, así como las planicies situadas entre Jaén y Huarango. Aunque estos bosques están muy alterados por el uso ganadero y agrícola del suelo, podemos observar un dosel arbóreo de entre 15 y 20 m de altura, donde Hura crepitans indica una cierta humedad edáfica y resistencia a las inundaciones. En sus espacios más alterados por la ganadería podemos destacar los pastizales con Hyparrhenia rufa (Nees) Stapf, donde interviene también Dalechampia aristolochiifolia Kunth (fig. 1a). 


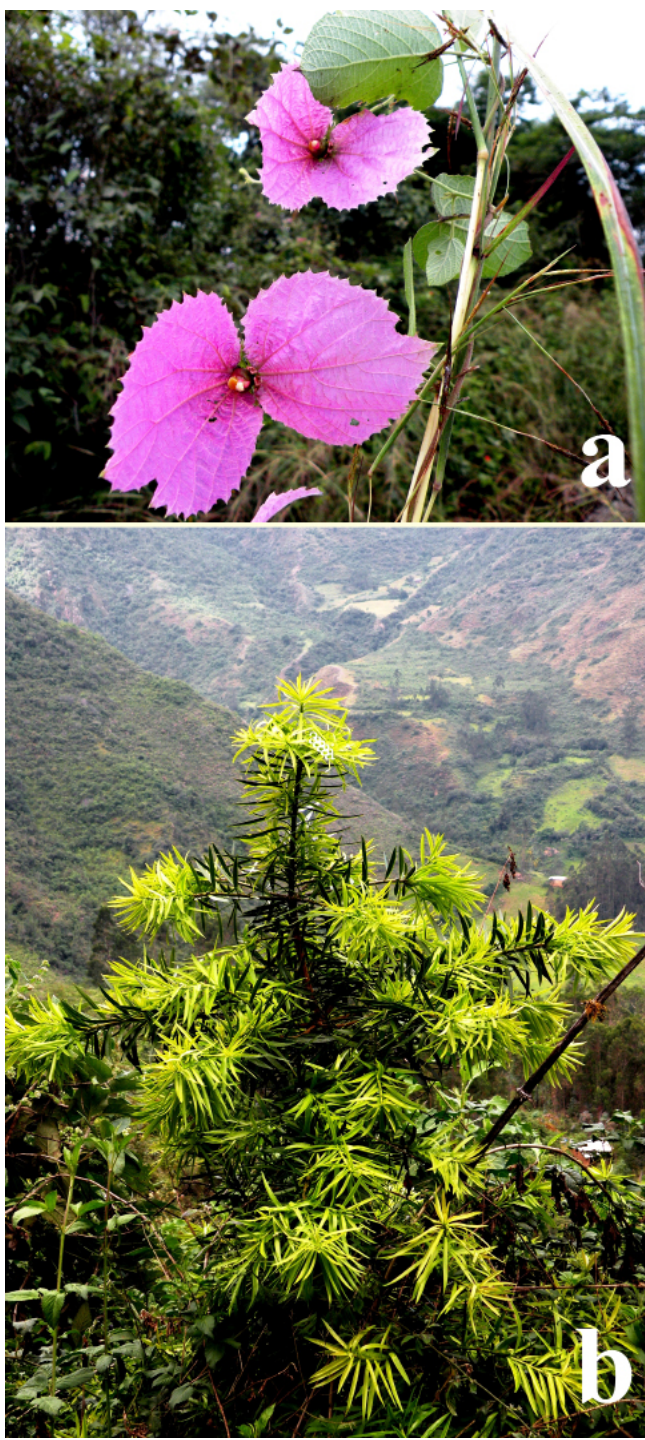

Figura 1. a- Dalechampia aristolochiifolia con Hyparrhenia rufa en un claro del bosque de Muntingia calabura y Hura crepitans en las proximidades de Huarango, b- Especimen joven de Podocarpus oleifolius; detrás el bosque de Axinaeo nitidae-Podocarpetum oleifolii en Cachil. a-Dalechampia aristolochiifolia with Hyparrhenia rufa in a clearing of the Muntingia calabura and Hura crepitans forest near Huarango, b- Young specimen of Podocarpus oleifolius; behind the Axinaeo nitidae-Podocarpetum oleifolii in Cachil.
La presencia de Baccharis inamoena Gardner, Fuchsia mathewsii J.F. Macbr., Histiopteris incisa (Thunb.) J. Sm., Myriocarpa stipitata Benth. y Paullinia alata (Ruiz \& Pav.) G. Don en la tabla 2, nos llevan a incluir por el momento a esta comunidad en la clase Nectandro-Licarietea.

2. Los bosques lauroides y esclerófilos occidentales (Clase Pruno rigidaeOreopanacetea floribundi)

Axinaeo nitidae-Podocarpetum oleifolii Galán de Mera, Sánchez Vega, Montoya, Linares, Campos \& Vicente ass. nova [Tabla 3, holotypus ass. inv. 3, fig. 1b]

Especies características: Axinaea nitida Cogn., Chrysophyllum contumazense Sagást. \& M.O. Dillon, Miconia firma J.F. Macbr. y Podocarpus oleifolius D.Don.

Bosque lauroide mesotropical húmedohiperhúmedo, con una altura de hasta $40 \mathrm{~m}$, a veces con pendientes de hasta $30 \%$, orientadas hacia las perturbaciones pacíficas del SO. Se desarrollan sobre suelos profundos ácidos que provienen de materiales intrusivos.

$\mathrm{Su}$ composición florística presenta una buena cantidad de endemismos nor-peruanos (Axinaea nitida, Berberis buceronis J.F. Macbr., Chrysophyllum contumacense, Miconia adinantha Wurdack, M. firma, Passiflora sagasteguii Skrabal \& Weigend y Symplocos sandemanii B. Ståhl) y ecuato-peruanos (Fuchsia ayavacensis Kunth, Ilex uniflora Benth., Oreopanax eriocephalus Harms y Viola arguta Willd. ex Roem. \& Schult.), aunque algunas especies también alcanzan los bosques húmedos montanos de Colombia (Delostoma integrifolium D. Don, Miconia denticulata Naudin y Monnina pilosa Kunth). El resto presenta una distribución muy amplia, sobre todo en los Andes orientales. La especie directriz es Podocarpus oleifolius cuyo límite andino meridional oriental está en el departamento de Cochabamba (Bolivia), y el occidental está precisamente en las localidades inventariadas de Contumazá (Cajamarca) 
Tabla 2

1-6: Comunidad de Cecropia montana y Heliocarpus americanus

7: Comunidad de Muntingia calabura y Hura crepitans

(Nectandro-Licarietea, Nectandro-Clusietalia, Tibouchino-Vismion)

N. de inventario

Altitud (m)

$\begin{array}{lllllll}1643 & 1689 & 1645 & 1776 & 1764 & 1764 & 575\end{array}$

Área $\left(\mathrm{m}^{2}\right)$ 500

Pendiente

500
500

500 500

$500 \quad 500 \quad 500$

Orientación

$30 \quad 40$

Plantas de comunidades

Heliocarpus americanus

Cecropia montana

NO NO

$\stackrel{2}{0}$
0
-
-
-
-

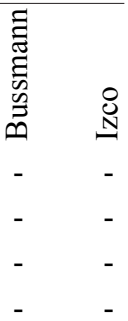

Piper nudilimbum

Muntingia calabura

Hura crepitans

Albizia multiflora

Leucaena trichodes

Salvia hirta

\section{Endemismos}

Fuchsia mathewsii

Anthurium hamiltonii

Miconia adinantha

Chusquea polyclados

$\begin{array}{llllllllll}2 & . & . & 4 & 4 & 4 & . & . & * * & . \\ 5 & . & 4 & . & . & . & . & . & * * & * * \\ . & . & . & . & . & . & 2 & * & * & . \\ . & . & . & . & . & . & 3 & . & * * & . \\ . & . & . & . & . & . & 4 & . & . & . \\ . & . & . & . & . & . & 1 & . & . & . \\ . & . & . & . & . & . & 1 & . & . & . \\ . & . & . & . & . & . & + & . & . & .\end{array}$

Características de alianza

Weinmannia spruceana

Cinchona pubescens

Isertia laevis

Piper lineatum

Clusia crenata

Myrsine latifolia

Mikania decora

Características de orden y clase

Siparuna aspera

Baccharis inamoena

Viburnum reticulatum

Histiopteris incisa

Cyathea caracasana

Smilax domingensis

Lindsaea arcuata

$\begin{array}{lllll}+ & 1 & 1 & 1 & 2 \\ 1 & 2 & . & 1 & 1 \\ . & 1 & 2 & 1 & + \\ 1 & . & 1 & 1 & 1 \\ . & . & . & 1 & 1 \\ 1 & . & . & 1 & . \\ 1 & 2 & 1 & . & .\end{array}$


Condaminea corymbosa

Pouzolzia poeppigiana

Alchornea glandulosa

Psychotria buchtienii

Paullinia alata

Rubus urticifolius

Mandevilla fragrans

Myriocarpa stipitata

Anemia phyllitidis

Psammisia coarctata

\section{Compañeras}

Pteridium arachnoideum

Pennisetum peruvianum

Pitcairnia riparia

Peperomia trinervis

Jatropha sp.

Olyra latifolia

Nephrolepis cordifolia

Anthurium giganteum

Anthurium trilobum

Piper peltatum

Seemannia sylvatica

Begonia peruviana

Cyrtocymura scorpioides

Gleichenella pectinata

Tradescantia cymbispatha

Thelypteris leprieurii

Elaphoglossum cuspidatum

Serpocaulon caceresii

Hypolepis parallelogramma

Solanum sp.

Ditassa endoleuca

Ichnanthus nemorosus

Ruellia brevifolia

Otras compañeras. En 1: Adiantum macrophyllum 1. En 5: Phragmipedium boissierianum 1. En 6: Aspilia jelskii + , Ipomoea sp. 1.

Localidades. 1-6- Cajamarca, San Ignacio, La Coipa (1- 17M 0729972-9404867, 2- 17M 07300119404994, 3- 17M 0729929-9404872, 4- 17M 0728018-9404978, 5-17M 0728904-9405322, 6- 17M 0728904-9405322, 7- 17M 0724348-9394688), 8- Cajamarca, San Ignacio, Tamborapa (17M 07243489394688). Géneros $(*)$ y especies (**) también presentes en Gentry (2002), Bussmann (2003) e Izco (2013). Genera (*) and species (**) also present in Gentry (2002), Bussmann (2003) and Izco (2013). 
(Vicuña-Miñano, 2005).

La distribución de esta nueva asociación es el SO del departamento de Cajamarca, con los bosques de Cachil y Tongod, en la provincia de San Miguel (Vicuña-Miñano, 2005).

Verbesino auriculigerae-Siparunetum muricatae Galán de Mera, Sánchez Vega, Montoya, Linares, Campos \& Vicente ass. nova [Tabla 3, holotypus ass. inv. 7]

Especies características: Axinaea nitida, Siparuna muricata (Ruiz \& Pav.) A. DC. y Verbesina auriculigera S.F. Blake.

Bosque lauroide mesotropical, húmedohiperhúmedo que alcanza un dosel de $20 \mathrm{~m}$. Se trata de una comunidad derivada de la acción antrópica con árboles de tronco de unos 20 cm de ancho, que sustituye a los bosques de Podocarpus L'Hér. ex Pers.

Como en la asociación anterior el número de endemismos, circunscritos a los departamentos del N del Perú entre San Martín, Lambayeque, Piura y Cajamarca, es muy elevado (Axinaea nitida, Berberis buceronis, Passiflora sagasteguii, Siphocampylus macropodoides Zahlbr., Symplocos sandemanii y Verbesina auriculigera), lo que indica una distribución amplia de la asociación. Sin embargo, Siparuna muricata se extiende desde los bosques húmedos de Ecuador a Bolivia, lo que prevé un número elevado de asociaciones por describir.

\section{Berberido beauverdianae-Myrcianthetum} myrsinoidis Galán de Mera, Sánchez Vega, Montoya, Linares, Campos \& Vicente ass. nova [Tabla 4, holotypus ass. inv. 4]

Especies características: Berberis beauverdiana C.K. Schneid, Lepechinia mollis (Epling) Epling y Myrcianthes myrsinioides (Kunth) Grifo.

Bosque esclerófilo mesotropical inferior seco-subhúmedo, donde los árboles alcanzan una altura de hasta $10 \mathrm{~m}$ sobre suelos ácidos derivados de granitos y granodioritas, y siempre en pendientes (20-40\%) de orientación S-SO, que están expuestas a las lluvias originadas por la corriente cálida ecuatorial y las nieblas ascendentes que, procedentes del Océano Pacífico, originan criptoprecipitaciones.

Catenalmente se muestran en contacto, a mayor altitud y menor humedad, con los bosques de Podocarpus oleifolius.

Berberido-Myrcianthetum incluye un menor número de endemismos que AxinaeoPodocarpetum, siendo su distribución más amplia en el sur-occidente del departamento de Cajamarca, desde Contumazá a la región de Querocoto-Querocotillo. Aunque nuestros inventarios no recogen esta última región, el transecto de Gentry (1995-2015) en El Pargo incluye bastantes elementos comunes, como Clusia multiflora Kunth, Hesperomeles lanuginosa Ruiz \& Pav. ex Hook., Myrsine coriacea (Sw.) R. Br. ex Roem. \& Schult. y Oreocallis grandiflora (Lam.) R.Br.

\section{Aristeguietio discoloris-Kageneckietum} lanceolatae Galán de Mera, Sánchez Vega, Montoya, Linares, Campos \& Vicente ass. nova [Tabla 5, holotypus ass. inv. 1]

Especies características: Aristeguietia discolor (DC.) R.M. King \& H. Rob., Baccharis emarginata (Ruiz \& Pav.) Pers., Calceolaria rugulosa Edwin, Colletia spinosissima J.F. Gmel. y Kageneckia lanceolata Ruiz \& Pav.

Bosque achaparrado esclerófilo mesotropical superior seco-subhúmedo, de unos $5 \mathrm{~m}$ de altura, que se asienta sobre pendientes de hasta $40 \%$, muy pedregosas con suelos ácidos de origen granítico. Igual que en las asociaciones anteriores este bosque aparece orientado hacia el S-SO, expuesto a la humedad del Océano Pacífico.

Aunque nuestros inventarios proceden de la región de Contumazá, al SO del departamento de Cajamarca, sus especies características llegan hasta el bosque de Kañaris (Lambayeque) (Llatas Quirós \& López-Mesones, 2005) al S de la depresión de Huancabamba.

Aristeguietio-Kageneckietum se diferencia 
por la riqueza de elementos andinos comunes con Bolivia y Argentina que alcanzan en estos bosques su límite septentrional occidental (Ageratina sternbergiana (DC.) R.M. King \& H. Rob., Brachyotum naudinii Triana, Clematis haenkeana C. Presl, Eryngium weberbaueri H. Wolff, Kageneckia lanceolata, Mutisia acuminata Ruiz \& Pav., Viguiera procumbens (Pers.) S.F. Blake). La orla del bosque, al ser menos umbrío, se enriquece con elementos de la clase Clematido peruvianae-Baccharitetea latifoliae (Baccharis latifolia (Ruiz \& Pav.) Pers., Muehlenbeckia tamnifolia (Kunth) Meisn., Mutisia acuminata, Ophryosporus chilca (Kunth) Hieron., Otholobium munyense (J.F. Macbr.) J.W. Grimes, Salvia oppositiflora Ruiz \& Pav. y Viguiera procumbens).

Aristeguietia discolor, que hemos elegido como característica de la asociación por su fidelidad, también está presente en las arbustedas supratropicales seco-subhúmedas centroperuanas de la asociación Aristeguietio discoloris-Baccharidetum latifoliae (Galán de Mera et al., 2004).

Monnino pilosae-Myrcianthion myrsinioidis Galán de Mera, Sánchez Vega, Montoya, Linares, Campos \& Vicente all. nova

[Holotypus all.: Berberido beauverdianaeMyrcianthetum myrsinoidis Galán de Mera, Sánchez Vega, Montoya, Linares, Campos \& Vicente ass. nova]

Especies características: Aphelandra acanthifolia Hook., Berberis buceronis, B. jelskiana C.K. Schneid., B. pichinchensis Turcz., Delostoma integrifolium, Fuchsia ayavacensis, Ilex uniflora, Maytenus boarioides Loes., Miconia denticulata, Monnina pilosa, Myrcianthes myrsinoides, Oreopanax eriocephalus, Passiflora sagasteguii, Scutellaria scutellarioides (Kunth) Harley y Viburnum mathewsii (Oerst.) Killip \& A.C. Sm.

Alianza que reúne a los bosques desde secos a hiperhúmedos asentados al occidente de los departamentos de Cajamarca, La Libertad, Lambayeque y Piura, entre el río Chicama y la depresión de Huancabamba. Desde el punto de vista florístico se caracterizan por el elevado número de endemismos nor-peruanos (Axinaea nitida, Begonia polypetala A.DC., Berberis buceronis, Chrysophyllum contumazense, Cronquistianthus marrubiifolius (Hieron.) R.M. King \& H. Rob., Lepechinia mollis, Munnozia ferreyrii H. Rob., Passiflora sagasteguii, Ribes colandina Weigend, Symplocos sandemanii, Siphocampylus macropodoides y Urtica peruviana Geltman) y de especies comunes con Ecuador (Alternanthera villosa Kunth, Aphelandra acanthifolia, Baccharis emarginata, Begonia acerifolia Kunth, Berberis beauverdiana, B. jelskiana, Calceolaria calycina Benth., C. rugulosa, Cordia krauseana Killip, Croton abutiloides Kunth, Fuchsia ayavacensis, Geranium chilloense Willd. ex Kunth, Ilex uniflora, Lamourouxia sylvatica Kunth, Lepechinia lamiifolia (Benth.) Epling, Maytenus boarioides, Ophryosporus chilca, Oreocallis grandiflora, Oreopanax eriocephalus, Phenax laxiflorus Wedd., Solanum barbulatum Zahlbr., Viburnum mathewsii y Viola arguta) y Colombia (Berberis pichinchensis Turcz., Delostoma integrifolium, Duranta obtusifolia Kunth, Monnina pilosa, Poa aequatoriensis Hack. y Scutellaria scutellarioides (Kunth) Harley) (fig. 2), aunque al ascender al piso mesotropical superior, con la asociación AristeguietioKageneckietum, se hace evidente el elemento centro- y sur-andino, con plantas también presentes en Bolivia (Ageratina sternbergiana, Brachyotum naudinii, Eryngium weberbaueri, Polystichum nudicaule Rosenst. y Viguiera procumbens) y Argentina (Clematis haenkeana, Colletia spinosissima, Coreopsis fasciculata Wedd., Kageneckia lanceolata y Mutisia acuminata). La alteración de los bosques de esta alianza mediante quemas favorecidas para el pastoreo vacuno y caprino conduce a pastizales presididos por Paspalum candidum (Flüggé) Kunth y Poa aequatoriensis.

Por su composición florística, incluimos a 
Tabla 3

1-5: Axinaeo nitidae-Podocarpetum oleifolii Galán, Sánchez, Montoya, Linares, Campos \& Vicente ass. nova:

6-8: Verbersino auriculigerae-Siparunetum muricatae Galán, Sánchez, Montoya, Linares, Campos \& Vicente ass. nova (Pruno-Oreopanacetea, Cestro-Prunetalia, Monnino-Mycianthion)

N. de inventario

Altitud (m)

Área $\left(\mathrm{m}^{2}\right)$

Pendiente $(\%)$

Orientación

Características de asociaciones

Podocarpus oleifolius

Axinaea nitida

Chrysophyllum contumazense

Miconia firma

Siparuna muricata

Verbesina auriculigera

Características de alianza

Miconia denticulata

Ilex uniflora

Passiflora sagasteguii

Fuchsia ayavacensis

Aphelandra acanthifolia

Delostoma integrifolium

Oreopanax eriocephalus

Monnina pilosa

Myrcianthes myrsinoides

Berberis buceronis

Características de orden y clase

Blechnum occidentale

Trichilia tomentosa

Chusquea scandens

Clusia multiflora

Myrsine latifolia

Palicourea amethystina

Solanum maturecalvans

Hedyosmum scabrum

Solanum oblongifolium

Cervantesia tomentosa $\begin{array}{lllllllll}1 & 2 & 3 & 4 & 5 & 6 & 7 & 8 & \stackrel{0}{0} \text { 员 }\end{array}$ $\begin{array}{llllllll}2529 & 2518 & 2512 & 2517 & 2520 & 2699 & 2629 & 2830\end{array}$

$\begin{array}{llllllll}500 & 500 & 500 & 500 & 500 & 500 & 500 & 500\end{array}$

$20 \quad 30$

SO SO

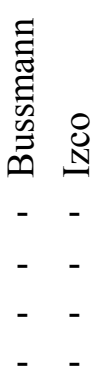


Clusia pseudomangle

Miconia adinantha

Vallea stipularis

Oreocallis grandiflora

Meliosma frondosa

Piper barbatum

Saurauia peruviana

Mauria heterophylla

Brachyotum naudinii

Rhamnus sphaerosperma

Scutia spicata

Phenax laxiflorus

\section{Compañeras}

Citharexylum flexuosum

Cronquistianthus marrubiifolius

Ageratina sternbergiana

Oreopanax aff. cuspidatus

Adiantum concinnum

Rubus robustus

Dryopteris cf. wallichiana

Calceolaria calycina

Solanum caripense

Valeriana $s p$.

Viola arguta

Lupinus mutabilis

Munnozia ferreyrii

Pteris muricata

Niphidium crassifolium

Pteridium arachnoideum

Aphelandra eurystoma

Mikania sp.

Geranium chilloense

Symplocos sandemanii

Siphocampylus macropodoides

Begonia acerifolia

Otras compañeras. En 1: Solanum barbulatum 1, Phyllanthus niruri 1. En 3: Liabum solidagineum 1, Tecoma stans + , Tradescantia cymbispatha 1, Croton abutiloides + . En 4: Smilax sp. +, Urtica peruviana +, En 6: Wedelia latifolia 1, Paspalum candidum 1, Rubus sp. 1, Fabaceae +. En 8: Luffa operculata 1, Monactis flaverioides 1, Mutisia acuminata 3, Oxalis peduncularis 1, Lepechinia lamiifolia + , Vicia andina 1, Pappobolus sp. + , Salvia punctata + .

Localidades. 1-7- Cajamarca, Contumazá, Bosque de Cachil (1- 17M 0745245-9181860, 2- 17M 0745310-9181806, 3- 17M 0745412-9181644, 4- 17M 0745479-9181568, 5- 17M 0745377-9181032, 6- 17M 0743416-9181120, 7- 17M 0744375-9180906), 8- Cajamarca, Contumazá, bajada a Guzmango (17M 0737789-9184998). Géneros $\left(^{*}\right)$ y especies $(* *)$ también presentes en Bussmann (2003) e Izco (2013). Genera (*) and species (**) also present in Bussmann (2003) and Izco (2013). 


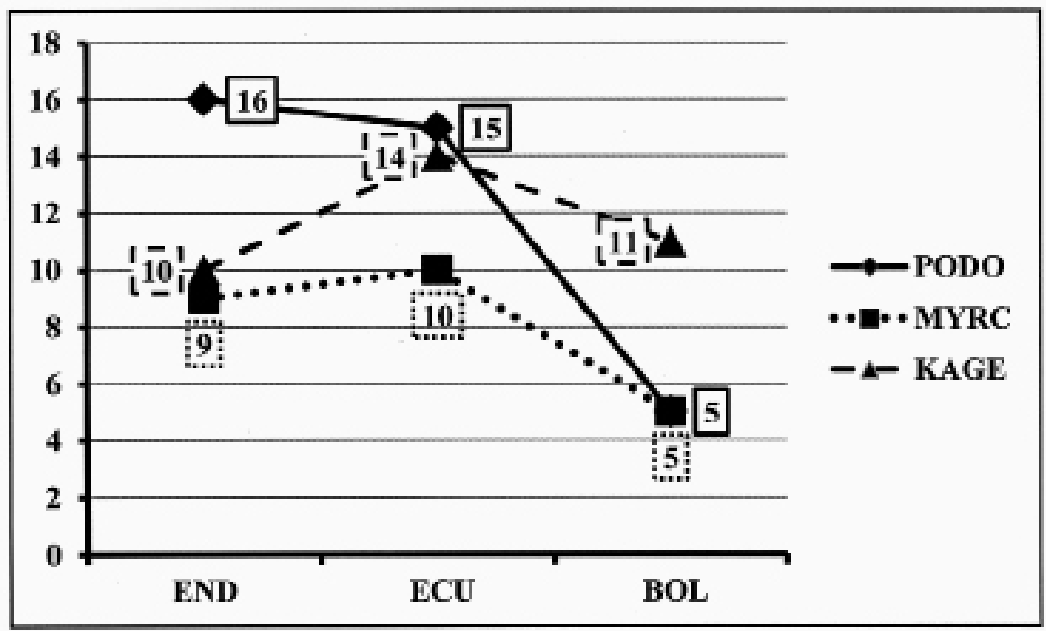

Figura 2. Variabilidad en el número de endemismos (END), de especies comunes con Ecuador y Colombia (ECU) y de especies comunes con Bolivia y Argentina (BOL), de los inventarios levantados de las asociaciones Axinaeo nitidae-Podocarpetum oleifolii (PODO), Berberido beauverdianae-Myrcianthetum myrsinoidis (MYRC), y Aristeguietio discoloris-Kageneckietum lanceolatae (KAGE). Variability of endemism number (END), common species with Ecuador and Colombia (ECU), and with Bolivia and Argentina (BOL), in the plots studied of Axinaeo nitidae-Podocarpetum oleifolii (PODO), Berberido beauverdianae-Myrcianthetum myrsinoidis (MYRC), and Aristeguietio discoloris-Kageneckietum lanceolatae (KAGE) associations.

los bosques lauroides andinos occidentales en el orden Cestro auriculati-Prunetalia rigidae (Galán de Mera et al., 2002) y en la clase Pruno rigidae-Oreopanacetea floribundi (Galán de Mera, 2005).

Monnino-Myrcianthion es vicariante de Myrcianthion quinquelobae (Galán de Mera et al., 2002) que reúne a los bosques lauroides relictos occidentales del departamento de Lima. La clase Pruno-Oreopanacetea también está representada en Ecuador a través de la alianza Oreopanacion floribundi (Lauer et al., 2001), y parece alcanzar la franja altoandina sobre los $3000 \mathrm{~m}$ en la Cordillera Central de Colombia (Van der Hammen, 2003; Rangel, 2015).

3. Las arbustedas heliófilas (Clase Clematido peruvianae-Baccharitetea latifoliae)

Baccharito latifoliae-Monactinetum flaverioidis Galán de Mera, Sánchez Vega, Montoya, Linares, Campos \& Vicente ass. nova [Tabla 6, holotypus ass. inv. 2]

Especies características: Baccharis latifolia,
Calceolaria triloba Edwin, Cronquistianthus glomeratus (DC.) R.M. King \& H. Rob., Monactis flaverioides Kunth y Siphocampylus albus E. Wimm.

Arbustedas heliófilas supra-mesotropicales subhúmedas-secas, cuya especie directriz es el endemismo nor-peruano Monactis flaverioides. Representan la orla boscosa o primera etapa de sustitución de las alisedas de la asociación Valleo stipularis-Alnetum acuminatae (Galán de Mera et al., 2002) y de los queñuales de la asociación Barnadesio dombeyanaePolylepidetum racemosae (Galán de Mera et al., 2013). La quema de estas arbustedas cuando se encuentran en suelos arcillosos con compensación edáfica propios de las alisedas, favorecen el pastizal autóctono de Andropogon lateralis Nees, fácil de encontrar en los márgenes de los cultivos. En cambio, si los pastos son frecuentados por la ganadería vacuna, estos acaban enriqueciéndose con plantas introducidas, como Lolium multiflorum Lam., Medicago lupulina L. o Trifolium pratense L. 
Si la asociación Baccharito-Monactinetum se asienta sobre los cambisoles de origen granítico del dominio de los queñuales, los pastos de contacto son los de la jalca supratropical con Hypericum laricifolium Juss.

Monactino flaverioidis-Colignonietum parviflorae Galán de Mera, Sánchez Vega, Montoya, Linares, Campos \& Vicente ass. nova prov. [Tabla 6]

Especies características: Alternanthera villosa, Colignonia parviflora (Kunth) Choisy, Monactis flaverioides y Verbesina auriculigera.

Asociación mesotropical húmeda donde el elemento andino oriental Colignonia parviflora es abundante en zonas perturbadas, y se hace notorio a través de sus brácteas blanquecinas. En el territorio estudiado forma la orla y primera etapa de sustitución de los bosques de Verbesino auriculigerae-Siparunetum muricatae.

Otholobio munyensis-Rubion robusti Galán de Mera, Sánchez Vega, Montoya, Linares, Campos \& Vicente all. nov.

[Holotypus all.: Baccharito latifoliaeMonactinetum flaverioidis Galán de Mera, Sánchez Vega, Montoya, Linares, Campos \& Vicente ass. nova]

Especies características: Ageratina fastigiata (DC.) R.M. King \& H. Rob., A. sternbergiana, Calceolaria calycina, Colignonia parviflora, Otholobium munyense, Rubus robustus C. Presl y Salvia alborosea Epling \& Játiva.

Es la alianza que reúne a las arbustedas heliófilas meso- y supratropicales subhúmedohúmedas del N del Perú, Ecuador y ceja de selva oriental peruana, donde al menos Colignonia parviflora, Otholobium munyense y Rubus robustus también están representadas.

Otholobio-Rubion es vicariante de la alianza centro- y sur-peruana Mutisio acuminataeOphryosporion peruvianae (Galán de Mera et al., 2002) y Saturejion bolivianae del oriente boliviano (Seibert \& Menhofer, 1991). Todas se engloban en el orden Mutisio acuminataeBaccharitetalia latifoliae (Galán de Mera et al., 2002).

\section{Clematido peruvianae-Baccharitetea latifoliae}

Galán de Mera, Sánchez Vega, Montoya, Linares, Campos \& Vicente cl. nova

[Sin.: Baccharitetea Lauer, Rafiqpoor \& Theisen 2001, nom. inv. (art. 5 y 17); holotypus cl.: Mutisio acuminatae-Baccharitetalia latifoliae Galán de Mera \& Cáceres in Galán de Mera, Rosa \& Cáceres 2002]

Es pecies caracterís ti c a s: Austrocylindropuntia subulata (Muehlenpf.) Backeb., Baccharis latifolia, Calceolaria bicrenata Ruiz \& Pav., Cantua buxifolia Juss. ex Lam., Clematis haenkeana, C. peruviana DC., Cynanchum longirostrum (K. Schum.) W.D. Stevens, Lupinus mutabilis Sweet, Minthostachys spicata (Benth.) Epling, $M$. mollis (Benth.) Griseb., Muehlenbeckia hastulata (Sm.) I.M. Johnst., Mutisia acuminata, Passiflora tarminiana Coppens \& V.E. Barney, Proustia cuneifolia D. Don, Rubus acanthophyllos Focke, R. robustus, Stevia mandonii Sch.Bip. ex B.L.Rob., Tagetes elliptica $\mathrm{Sm}$. y Viguiera procumbens.

Clase que reúne a las arbustedas heliófilas andinas que forman un manto externo o etapa de sustitución de alisedas, queñuales, bosques orientales de ceja de selva, y bosques húmedos andinos en general, aunque también se pueden presentar como comunidades climácicas sobre suelos con elevada pedregosidad, zonas que han sufrido avalanchas, o cuando el ombroclima es semiárido o seco. Además aparecen sus especies características en los márgenes de cultivos o proximidades de las poblaciones. Entre ellas cabe destacar a Lupinus mutabilis, que se comporta como una especie pionera en la regeneración de las arbustedas.

4. Los pajonales del páramo o jalca peruana (Clase Calamagrostietea vicunarum)

Calamagrostio tarmensis-Hypericetum 
Tabla 4

Berberido beauverdianae-Myrcianthetum myrsinoidis Galán, Sánchez, Montoya, Linares, Campos \& Vicente ass. nova

(Pruno-Oreopanacetea, Cestro-Prunetalia, Monnino-Mycianthion)

N. de inventario

Altitud (m)

Área $\left(\mathrm{m}^{2}\right)$

Pendiente (\%)

Orientación

\begin{tabular}{|c|c|c|c|c|c|c|c|}
\hline 1 & 2 & 3 & 4 & 5 & $\stackrel{\vec{E}}{\stackrel{\Xi}{0}}$ & 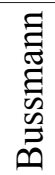 & $\begin{array}{l}\text { D } \\
\text { N }\end{array}$ \\
\hline 2661 & 2525 & 2555 & 2517 & 2440 & - & - & - \\
\hline 500 & 500 & 500 & 500 & 500 & - & - & - \\
\hline 20 & 40 & 30 & 40 & 20 & - & - & - \\
\hline SO & $\mathrm{O}$ & SO & $\mathrm{S}$ & SO & - & - & - \\
\hline
\end{tabular}

Características de asociación y alianza

Myrcianthes myrsinoides

Berberis beauverdiana

Oreopanax eriocephalus

Monnina pilosa

Miconia denticulata

Ilex uniflora

Viburnum mathewsii

Passiflora sagasteguii

Aphelandra acanthifolia

Maytenus boarioides

Lepechinia mollis

Características de orden y clase

Myrsine latifolia

Siparuna muricata

Clusia multiflora

Mauria heterophylla

Oreocallis grandiflora

Hesperomeles lanuginosa

Prunus rigida

Myrsine coriacea

Clematis haenkeana

Rhamnus sphaerocarpa

Podocarpus oleifolius

Verbesina auriculigera

$\begin{array}{cccccccc}4 & 4 & 4 & 4 & 4 & . & . & . \\ 1 & 1 & + & 1 & 1 & . & * * & * * \\ 2 & 1 & 1 & 1 & 1 & * & * & . \\ 1 & 1 & + & 1 & 1 & . & * * & . \\ 2 & . & 1 & + & 1 & * & * & * \\ 1 & . & 1 & 2 & 1 & . & * & * \\ + & . & 1 & 1 & . & * & * * & . \\ . & 1 & + & 1 & + & . & * & . \\ . & 1 & 1 & . & 1 & . & * & . \\ + & . & + & 1 & + & . & . & . \\ 1 & 1 & . & . & . & . & . & .\end{array}$

Acca macrostema 


\section{Compañeras}

Rubus robustus

Pteridium arachnoideum

Cronquistianthus marrubiifolius

Citharexylum flexuosum

Adiantum concinnum

Oxalis peduncularis

Croton abutiloides

Blechnum occidentale

Ageratina sternbergiana

Valeriana $s p$.

Liabum solidagineum

Solanum caripense

Begonia polypetala

Phoradendron nervosum

Oreopanax aff. cuspidatus

Salvia oppositiflora

Thalictrum longistylum

Monactis flaverioides

Niphidium crassifolium

Bomarea cornuta

Bidens squarrosa

Tecoma stans

Otras compañeras. En 1: Stevia caracasana + . En 2: Alternanthera villosa 1, Anchietea peruviana.+

Localidades. 1-2- Cajamarca, Contumazá, Bosque de Cachil (1- 17M 0743503-9181020), 3Cajamarca, Trinidad (17M 0724182-9191745), 4- La Libertad, Cascas (17M 0750051-9175238), 5Cajamarca, San Miguel de Pallaqués (17M 0713476-9242387). Géneros $\left(^{*}\right)$ y especies $(* *)$ también presentes en Gentry (2002), Bussmann (2003) e Izco (2013). Genera (*) and species (**) also present in Gentry (2002), Bussmann (2003) and Izco (2013).

laricifolii Galán de Mera, Sánchez Vega, Montoya, Linares, Campos \& Vicente ass. nova [Tabla 7, holotypus ass. inv. 1]

Especies características: Calamagrostis tarmensis Pilg., Calceolaria cajabambae Kraenzl., Geranium peruvianum Hieron., Hieracium peruanum Fr., Hypericum laricifolium, Jungia stuebelii (Hieron.) Crisci, Muhlenbergia caxamarcensis Laegaard \& Sánchez Vega, Paranephelius ferreyrii H.Rob. y Tridax peruviensis A.M.Powell.

Pajonal supratropical húmedo, muy rico en endemismos nor-peruanos (Calceolaria cajabambae, Geranium peruvianum, Muhlenbergia caxamarcensis, Paranephelius ferreyrii y Tridax peruviensis) y de amplia distribución (Dioscorea ancashensis R.Knuth, Festuca huamachucensis Infantes, Hieracium peruanum, Jungia stuebelii, Lupinus peruvianus y Stipa macbridei Hitchc.), que se asienta sobre 
suelos de tipo andosol derivados de sustratos graníticos, llegando a alcanzar $1 \mathrm{~m}$ de altura, y donde $H$. laricifolium normalmente sobresale por su altura y densidad. Desde el punto de vista dinámico son un estadio sucesional de los queñuales situados al S del departamento de Cajamarca, entre las cuencas de los ríos Socota y Moche, ya en el departamento de La Libertad. En la actualidad estos pastizales están volviendo a ser pastados por vicuñas que han sido recientemente reintroducidas como ganadería autóctona. Sin embargo, en otras muchas localidades son sustituidos por cultivos o especies introducidas (Lolium multiflorum, Medicago lupulina o Trifolium pratense) preferidas por la ganadería vacuna.

En áreas rocosas con escorrentía superficial, la asociación se enriquece con Cortaderia nitida (Kunth) Pilg. y Pitcairnia L'Hér. sp. 4282, pudiéndose describir la subasociación cortaderietosum nitidae Galán de Mera, Sánchez Vega, Montoya, Linares, Campos \& Vicente subass. nova (tab. 7, holotypus subass. inv. 10; especies características: Cortaderia nitida y Pitcairnia sp.).

Al $\mathrm{N}$ de la depresión de Huancabamba en este tipo de pajonales abundan las Ericáceas (Gaultheria buxifolia Willd., G. reticulata Kunth, Vaccinium crenatum (G.Don) Sleumer, $V$. floribundum Kunth) constituyendo la asociación vicariante Oreobolo goeppingeri-Hypericetum laricifolii Sabogal 2014 (lectotypus ass. tab. 10, inv. 9: Hypericum laricifolium 2, Gaultheria buxifolia +, Sphagnum L. sp. 1 4, Gentianella bicolor (Wedd.) Fabris ex J.S.Pringle r, Baccharis latifolia 1, Cyperaceae Juss. 2, Oreobolus goeppingeri Suess. 1, Vaccinium floribundum 1, Blechnum L. sp. 1, Senecio L. sp. r, Aciachne pulvinata Benth. 1, Valeriana microphylla Kunth 2, Scirpus cf. caespitosus Willd. ex Kunth 1 (transcripción del original); especies características: Chaptalia oblonga D.Don, Gaultheria buxifolia, G. reticulata, Hypericum laricifolium, Loricaria ferruginea (Ruiz \& Pav.) Wedd., Lycopodium clavatum
L., Vaccinium crenatum y V.floribundum). Esta comunidad también fue estudiada por Peyre (2015) aunque sin describir asociaciones ni unidades superiores.

\section{Agrostio tolucensis-Paspaletum bonplandiani}

Galán de Mera, Sánchez Vega, Montoya, Linares, Campos \& Vicente ass. nova [Tabla 8, holotypus ass. inv. 1]

Especies características: Agrostis tolucensis Kunth, Ascidiogyne sanchezvegae Cabrera, Paspalum bonplandianum Flüggé y Werneria stuebelii Hieron.

Pajonal climácico orotropical húmedohiperhúmedo que ocupa las cumbres del $\mathrm{N}$ del Perú sobre los $3800 \mathrm{~m}$, alcanzando una altura de unos $30 \mathrm{~cm}$. Se presenta sobre suelos profundos de tipo regosol derivados de roquedos graníticos, generalmente pedregosos con crioturbación.

En áreas con una cierta acumulación de agua y zonas con agua de escorrentía, la comunidad se enriquece con Calamagrostis rigescens (J.Presl) Scribn. y Puya fastuosa Mez, por lo que describimos la subasociación puyetosum fastuosae Galán de Mera, Sánchez Vega, Montoya, Linares, Campos \& Vicente (tab. 8, holotypus subass. inv. 4; especies características: Calamagrostis rigescens, Loricaria ferruginea, Lycopodium clavatum y Puya fastuosa). P. fastuosa es un endemismo del departamento de Cajamarca, del que solo conocemos registros en Coimolache (Mez, 1907) y Sallique (Marcelo Peña et al., 2006), aunque según el material fotográfico de Marcelo Peña \& Latorre Cuadros, 2010), tal vez esté también presente en las Lagunas Arrebiatadas, al N-O de la depresión de Huancabamba. Estas comunidades con $P$. fastuosa sobre suelos húmedos, son vicariantes de las de $P$. clava-herculis Mez \& Sodiro, Festuca subulifolia Benth. y Calamagrostis effusa (Kunth) Steud. de los alrededores de Quito (Ecuador) (Lauer et al., 2001), y de las de $P$. santosii Cuatrec. (Puyetum santosii) de 
Tabla 5

Aristeguietio discoloris-Kageneckietum lanceolatae Galán, Sánchez, Montoya, Linares, Campos \& Vicente ass. nova

(Pruno-Oreopanacetea, Cestro-Prunetalia, Monnino-Myrcianthion)

\begin{tabular}{|c|c|c|c|c|}
\hline N. inventario & 1 & 2 & 3 & 4 \\
\hline Altitud (m) & 3348 & 3351 & 3230 & 3180 \\
\hline Área $\left(\mathrm{m}^{2}\right)$ & 500 & 500 & 500 & 500 \\
\hline Pendiente $(\%)$ & 40 & 40 & 30 & 30 \\
\hline Orientación & SO & $\mathrm{SO}$ & $\mathrm{S}$ & $\mathrm{O}$ \\
\hline \multicolumn{5}{|c|}{ Características de asociación y alianza } \\
\hline Kageneckia lanceolata & 4 & 4 & 2 & 4 \\
\hline Aristeguietia discolor & 1 & 1 & 3 & 3 \\
\hline Baccharis emarginata & + & 2 & 4 & 3 \\
\hline Calceolaria rugulosa & 2 & 2 & 1 & 1 \\
\hline Colletia spinosissima & 3 & 2 & 3 & 2 \\
\hline Myrcianthes myrsinoides & 2 & 1 & . & 1 \\
\hline Salvia punctata & 1 & 2 & . & 1 \\
\hline Passiflora sagasteguii & 1 & 1 & . & 1 \\
\hline Monnina pilosa & 1 & 1 & . & . \\
\hline Scutellaria scutellarioides & 1 & . & 1 & . \\
\hline Lepechinia mollis & . & 2 & 2 & . \\
\hline Berberis pichinchensis & 1 & . & . & . \\
\hline Ribes colandina & 1 & . & . & . \\
\hline Maytenus boarioides & + & . & . & . \\
\hline Berberis jelskiana & . & . & 1 & . \\
\hline Calceolaria salicifolia & . & . & . & 1 \\
\hline Verbesina auriculigera & . & . & . & 1 \\
\hline \multicolumn{5}{|l|}{ Características de orden y clase } \\
\hline Mauria heterophylla & 1 & 2 & . & . \\
\hline Passiflora peduncularis & 1 & 1 & . & . \\
\hline Hesperomeles lanuginosa & . & 1 & . & 2 \\
\hline Oreopanax eriocephalus & . & . & + & 2 \\
\hline Cordia krauseana & 1 & 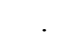 & . & . \\
\hline Clematis haenkeana & . & 2 & . & . \\
\hline Oreocallis grandiflora & . & . & 1 & 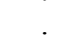 \\
\hline Duranta obtusifolia & . & . & . & 1 \\
\hline Oreopanax aff. cuspidatus & . & . & . & + \\
\hline Polystichum nudicaule & . & . & . & 1 \\
\hline \multicolumn{5}{|l|}{ Compañeras } \\
\hline Ageratina sternbergiana & 1 & + & 1 & 1 \\
\hline Cronquistianthus sp. & 2 & 2 & 2 & + \\
\hline Coreopsis fasciculata & 1 & . & 2 & 1 \\
\hline Cronquistianthus marrubiifolius & 1 & 1 & . & 1 \\
\hline Rubus robustus & . & 2 & 1 & 1 \\
\hline Eryngium weberbaueri & + & . & + & . \\
\hline Lupinus mutabilis & . & . & 1 & 1 \\
\hline
\end{tabular}

Otras compañeras. En 1: Vicia andina + , Lamorouxia sylvatica,+ Puya sp. + , Festuca sp. + . En 2: Ophryosporus chilca +, Clematis haenkeana 2, Muehlenbeckia tamnifolia 1, Pappophorum pappiferum +, Viguiera procumbens + , Baccharis latifolia 1, Otholobium munyense 1, Salvia oppositiflora 1, Mutisia acuminata 1. En 3: Festuca sp. 1, Bomarea sp. 1, Brachyotum naudinii +. En 4: Luffa operculata 1, Poa aequatoriensis +, Paspalum candidum 1, Nassella mucronata + , Nassella inconspicua + , Oreopanax aff. cuspidatus + , Valeriana sp. + .

Localidades. 1-4- Cajamarca, Contumazá, Bajada a Guzmango (1- 17M 0733474-9185952, 2- 17M 07335049185950, 3- 17M 0731669-9186090, 4- 17M 0732140-9185882). 
la Cordillera Oriental colombiana (Rangel et al., 1997).

Sin embargo, en el límite más meridional, la asociación adquiere plantas de ambientes más fríos y que significan una transición hacia la puna centroperuana. Con ellas describimos la subasociación calamagrostietosum curvulae Galán de Mera, Sánchez Vega, Montoya, Linares, Campos \& Vicente subass. nova [tab. 8, holotypus subass. inv. 5; especies características: Alchemilla frigida Wedd., Calamagrostis curvula (Wedd.) Pilg., Mniodes pulvinata Cuatrec. y Nassella depauperata (Pilg.) Barkworth].

\section{Agrostio tolucensis-Paspalion bonplandiani}

Galán de Mera, Sánchez Vega, Montoya, Linares, Campos \& Vicente all. nova

[Holotypus all.: Calamagrostio tarmensisHypericetum laricifolii Galán de Mera, Sánchez Vega, Montoya, Linares, Campos \& Vicente ass. nova]

Especies características: Agrostis boliviana Mez, A. tolucensis, Alchemilla frigida, Antennaria linearifolia Wedd., Aphanactis villosa S.F.Blake, Arcytophyllum filiforme (Ruiz \& Pav.) Standl., A. thymifolium (Ruiz \& Pav.) Standl., Bartsia adenophylla Molau, Belloa turneri Sagást. \& M.O.Dillon, Briza monandra (Hack.) Pilg., Calamagrostis tarmensis, Chrysactinium acaule (Kunth) Wedd., Festuca huamachucensis, Gaultheria glomerata (Cav.) Sleumer, Gentianella bicolor, Geranium multipartitum Benth., Hypericum aciculare Kunth, Lobelia tenera Kunth, Lupinus peruvianus Ulbr., Oritrophium peruvianum (Lam.) Cuatrec., Paspalum bonplandianum, Orthrosanthus chimboracensis (Kunth) Baker, Poa pauciflora Roem. \& Schult., Stevia andina B.L.Rob., Tridax tambensis Hieron. y Valeriana pilosa Ruiz \& Pav.

El territorio comprendido entre el departamento de Cajamarca y el centro de Ecuador, en la región del Chimborazo $\left(1^{\circ} \mathrm{de}\right.$ latitud N), apenas presenta altitudes sobre los
$4000 \mathrm{~m}$ (Lauer et al., 2001) lo que hace que la clase Calamagrostio effusae-Espeletietea grandiflorae Galán de Mera 2005 [Sin.: Espeletio-Calamagrostietea recto-effusae Cleef, Rangel \& Salamanca, Est. Ecos. Trop. 5: 17 (2003), nom. inv. art. 3g, art. 10] tenga su límite meridional en la cadena formada por los nevados Cotopaxi-Antisana, puesto que lo es también para Calamagrostis effusa (Jørgensen \& León-Yáñez, 1999; Tropicos, 2015). Para Espeletia Mutis ex Bonpl. el límite S está en la Cordillera de los Llanganates, donde crece E. pycnophylla subsp. llanganetensis Cuatrec. (Ulloa Ulloa \& Jørgensen, 2015). Estas altitudes inferiores del S de Ecuador y N de Perú hacen que no lleguen los elementos de CalamagrostioEspeletietea y sean sustituidos por plantas que tienen sobre todo una vocación andina oriental y se introducen desde Ecuador a Huánuco o Cusco, como Bartsia adenophylla, Hypericum aciculare, Lobelia tenera o Stevia andina. Todas ellas son características de la nueva alianza Agrostio-Paspalion, por el momento la única del nuevo orden Agrostio tolucensisPaspaletalia bonplandiani Galán de Mera, Sánchez Vega, Montoya, Linares, Campos \& Vicente ordo novo.

Asimismo, este orden, como indicamos en las tablas 7 y 8 , incluye a numerosas especies de la clase Calamagrostietea vicunarum (RivasMartínez \& Tovar, 1982; Gutte, 1985; Galán de Mera et al., 2014): Aciachne pulvinata, Alchemilla procumbens Rose, Baccharis caespitosa (Ruiz \& Pav.) Pers., Bromus lanatus Kunth, Calamagrostis curvula, Castilleja nubigena Kunth, Dioscorea ancashensis, Eryngium humile Cav., Hypericum silenoides Juss., Luzula racemosa Desv., Mniodes pulvinata, Muehlenbeckia volcanica (Benth.) Endl., Nassella depauperata, Novenia acaulis (Wedd. ex Benth. \& Hook.f.) S.E.Freire \& F. Hellwig, Oreithales integrifolia (DC.) Schltdl., Paranephelius ovatus A.Gray ex Wedd., P. uniflorus Poepp., Pernettya prostrata (Cav.) DC., Phyllactis rigida (Ruiz 
Tabla 6

1-7: Baccharito latifoliae-Monactinetum flaverioidis Galán, Sánchez, Montoya, Linares, Campos \& Vicente ass. nova; 8-9: Monactino flaverioidis-Colignonietum parviflorae Galán, Sánchez, Montoya, Linares, Campos \& Vicente ass. nova prov.

(Clematido-Baccharitetea, Mutisio-Baccharitetalia, Otholobio-Rubion)

\begin{tabular}{lcccccccccc}
\hline N. inventario & 1 & 2 & 3 & 4 & 5 & 6 & 7 & 8 & 9 & - - \\
Altitud $(\mathrm{m})$ & 3150 & 3150 & 3150 & 3163 & 3163 & 3523 & 3523 & 2661 & 2457 & - \\
Area $\left(\mathrm{m}^{2}\right)$ & 100 & 100 & 100 & 100 & 100 & 100 & 100 & 100 & 100 & - \\
Pendiente $(\%)$ & - & - & 30 & - & - & 50 & 50 & - & - & - \\
Orientación & - & - & $\mathrm{E}$ & - & - & $\mathrm{S}$ & $\mathrm{S}$ & - & - & -
\end{tabular}

\section{Características de asociaciones y alianza}

Monactis flaverioides

Baccharis latifolia

\begin{tabular}{|c|c|c|c|c|c|c|c|c|c|}
\hline 4 & 4 & 4 & 2 & 4 & 4 & 4 & 2 & 2 & \\
\hline 2 & 2 & 3 & 3 & 3 & 2 & 1 & & 1 & $* *$ \\
\hline v & . & . & . & . & r. & . & 4 & 1 & \\
\hline . & . & . & . & . & . & . & 2 & 4 & \\
\hline . & . & . & & & . & . & 1 & 1 & \\
\hline & & . & 2 & 2 & . & 1 & 2 & 2 & $* *$ \\
\hline 1 & 1 & 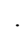 & 1 & . & . & . & 1 & 1 & \\
\hline 1 & 1 & 1 & 2 & . & . & . & . & & $*$ \\
\hline 1 & 1 & 1 & . & . & . & . & & & . \\
\hline 1 & 1 & . & 1 & . & . & . & & & . \\
\hline . & . & . & 1 & . & $\therefore$ & 2 & & & . \\
\hline . & . & . & . & . & 3 & 1 & & & * \\
\hline . & . & · & $\cdot$ & $\cdot$ & 1 & i & . & & $*$ \\
\hline$\cdot$ & ${ }^{\circ}$ & ${ }^{\circ}$ & 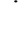 & . & . & & & & \\
\hline 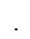 & 1 & 2 & 2 & 1 & . & . & . & & $*$ \\
\hline+ & . & + & 1 & & . & . & . & & $* *$ \\
\hline . & 1 & . & 1 & 2 & . & . & 1 & & $* *$ \\
\hline . & 1 & . & 2 & . & . & 1 & . & & $*$ \\
\hline . & . & . & 1 & . & . & . & 1 & & $*$ \\
\hline . & . & 1 & 1 & . & . & . & . & & . \\
\hline . & . & + & 1 & . & . & . & . & & . \\
\hline . & . & . & . & . & + & 2 & & & . \\
\hline . & . & . & . & . & . & . & . & 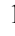 & . \\
\hline . & . & + & . & 1 & . & . & . & & $* *$ \\
\hline . & . & + & . & 1 & . & . & & & $*$ \\
\hline . & . & . & + & 1 & . & . & & & $* *$ \\
\hline . & . & . & . & 1 & + & . & . & & $*$ \\
\hline & . & . & . & . & + & 1 & & & $*$ \\
\hline+ & . & . & . & . & . & . & & & $*$ \\
\hline+ & . & . & . & 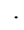 & . & . & & & $*$ \\
\hline 1 & . & . & . & + & . & . & & & . \\
\hline$\cdot$ & + & 1 & . & + & . & . & & & . \\
\hline . & . & 1 & . & 1 & . & . & & & ; \\
\hline . & . & 1 & . & & . & . & & & $\begin{array}{l}* \\
*\end{array}$ \\
\hline . & . & . & . & 1 & + & 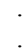 & & & $*$ \\
\hline . & . & . & . & . & $\begin{array}{l}+ \\
1\end{array}$ & $i$ & & & \\
\hline . & & 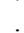 & & & & + & & & $* *$ \\
\hline
\end{tabular}

Colignonia parviflora

Verbesina auriculigera

Alternanthera villosa

Rubus robustus

Otholobium munyense

Salvia alborosea

Cronquistianthus glomeratus

Ageratina sternbergiana

Siphocampylus albus

Ageratina fastigiata

Calceolaria triloba

Calceolaria calycina

Características de orden y clase

Passiflora tarminiana

Minthostachys mollis

Clematis haenkeana

Calceolaria bicrenata

Viguiera procumbens

Tagetes elliptica

Cynanchum longirostrum

Rubus acanthophyllos

Lupinus mutabilis

\section{Compañeras}

Vallea stipularis

Jungia floribunda

Piper barbatum

Berberis jelskiana

Alonsoa linearis

Myrsine coriacea

Solanum sp.

Achyrocline alata

Senecio usgorensis

Myrsine pellucida

Barnadesia jelskii

Oreopanax eriocephalus

Calceolaria barbata

Polylepis racemosa

Bidens triplinervia

Otras compañeras. En 3: Paspalum sp. + , Calceolaria pavonii + , Scutellaria tomentosa + , Verbesina sanchezii + . En 6: Jaltomata sanchez-vegae 1. En 5: Chusquea polyclados +, Pecluma pectinata 1, Asplenium auriculatum 1. En 7: Smallanthus glabratus 2. En 8: Ilex uniflora 1, Niphidium crassifolium 1, Prunus rigida +, Rhamnus sphaerocarpa 1, Berberis beauvediana, 1. En 9: Heliotropium incanum 1, Urtica peruviana + . Bidens pilosa + , Alternanthera porrigens, 1.

Localidades. 1-5- Cajamarca, de Morocha a Bellavista (1- 17M 0766519-9253698, 2- 17M 0766546-9253642, 3- 17M 07665469253642, 4- 17M 0767084-9254170, 5- 17M 0767084-9254170), 6-7- Cajamarca, Hualgayoc (6- 17M 0765257-9251992, 7- 17M 0765257-9251992), 8-9- Cajamarca, Contumazá, junto al bosque de Cachil (8- 17M 0743503-9181020, 9- 17M 0743093-9179642). Géneros (*) y especies (**) también presentes en Lauer et al. (2001). Genera (*) and species (**) also present in Lauer et al. (2001). 
\& Pav.) Pers., Plantago sericea Ruiz \& Pav., Senecio tephrosioides Turcz., Stipa macbridei, Trichophorum rigidum (Steud.) Goetgh., Muasya \& D.A.Simpson, Werneria nubigena Kunth y $W$. villosa A.Gray. Por tanto, la clase Calamagrostietea vicunarum pasa a estar compuesta por los órdenes Calamagrostietalia vicunarum (Rivas-Martínez \& Tovar, 1982), Parastrephietalia quadrangularis (Galán de Mera et al., 2011) y Agrostio tolucensisPaspaletalia bonplandiani ordo novo.

\section{Vegetación y biogeografía}

En la figura 3 representamos el mapa de la vegetación potencial de Cajamarca, donde podemos observar bosques occidentales y orientales que perciben abundantes lluvias y nieblas, y con el páramo húmedo en las cumbres, localmente llamado jalca. Este esquema de vegetación se repite en los Andes ecuatorianos (Lauer et al., 2001) y colombianos (Rangel, 2015), con un subpáramo de bosques achaparrados, el páramo de gramíneas acompañadas de pequeños matorrales, caulirrósulas (Espeletia) y acantorrósulas (Puya Molina)(Cuatrecasas, 2003), y el superpáramo con gramíneas y pequeñas plantas arrosetadas (Van der Hammen, 2003), este último ausente en Perú.

En el departamento de Cajamarca, tanto los bosques húmedos como la jalca albergan una gran cantidad de endemismos; así, de las 2699 especies registradas (Marcelo Peña et al., 2006), 318 son angiospermas endémicas (Hensold, 1999). A esto hay que añadir que hay muchas especies recogidas en las tablas fitosociológicas que se introducen desde Colombia y Ecuador incluso hasta los Andes orientales, como Calceolaria calycina, Hypericum laricifolium, Ilex uniflora, Myrcianthes myrsinioides o Stevia

Figura 3 (página siguiente). Mapa de vegetación potencial de Cajamarca (Nomenclatura basada en el esquema sintaxonómico. Los números situados dentro del mapa son los de las estaciones meteorológicas de la tabla 1). Leyenda: 1-Comunidades infratropicales semiáridas de cactáceas (Armatocereo balsasensis-Cercidietum praecocis), 2- Bosques infratropicales seco-subhúmedos de Muntingia calabura y Hura crepitans, 3Bosques termotropicales subhúmedo-húmedos de Cecropia montana y Heliocarpus americanus, 4- Páramo supratropical húmedo del sector Loja-Cutervo (Oreobolo goeppingeri-Hypericetum laricifolii), 5- Bosques termotropicales secos orientales (Diplopterydo leiocarpae-Acacietum macracanthae), 6- Alisedas supramesotropicales seco-subhúmedas (Valleo stipularis-Alnetum acuminatae), 7- Páramo supratropical húmedo del sector Chota-Contumazá (Calamagrostio tarmensis-Hypericetum laricifolii) incluyendo bosques de Polylepis (Barnadesio dombeyanae-Polylepidetum racemosae) y arbustedas (Baccharito latifoliae-Monactinetum flaverioidis), 8- Páramo orotropical húmedo-hiperhúmedo (Agrostio tolucensis-Paspaletum bonplandiani), 9- Comunidades de cactáceas infratropicales arido-semiáridas occidentales (Loxopterygio huasanginisNeoraimondietum arequipensis), 10- Bosques termotropicales secos occidentales (Crotono ruiziani-Acacietum macracantae), 11- Bosques lauroides y esclerófilos mesotropicales de secos a hiperhúmedos occidentales (Axinaeo nitidae-Podocarpetum oleifolii, Verbesino auriculigerae-Siparunetum muricatae, Berberido beauverdianae-Myrcianthetum myrsinoidis, Aristeguietio discoloris-Kageneckietum lanceolatae). Potential vegetation map of Cajamarca (Vegetation names based on the syntaxonomical scheme. Numbers inside the map are the same that in the meteorological stations of the table 1). Legend: 1-Oriental infratropical semiarid cactus communities (Armatocereo balsasensis-Cercidietum praecocis), 2-Infratropical dry-subhumid forests of Muntingia calabura and Hura crepitans, 3- Thermotropical subhumid-humid forests of Cecropia montana and Heliocarpus americanus, 4-Supratropical humid paramo of the Loja-Cutervo sector (Oreobolo goeppingeri-Hypericetum laricifolii), 5- Oriental thermotropical dry forests (Diplopterydo leiocarpaeAcacietum macracanthae), 6- Supra-mesotropical dry-subhumid alder forests (Valleo stipularis-Alnetum acuminatae), 7- Supratropical humid páramo of the Chota-Contumazá sector (Calamagrostio tarmensisHypericetum laricifolii) including Polylepis forests (Barnadesio dombeyanae-Polylepidetum racemosae) and shrublands (Baccharito latifoliae-Monactinetum flaverioidis), 8-Orotropical humid-hyperhumid paramo (Agrostio tolucensis-Paspaletum bonplandiani), 9- Occidental infratropical arid-semiarid cactus communities (Loxopterygio huasanginis-Neoraimondietum arequipensis), 10-Occidental thermotropical dry forests (Crotono ruiziani-Acacietum macracantae), 11-Occidental mesotropical dry to hyperhumid laurel-like and sclerophyllous forests (Axinaeo nitidae-Podocarpetum oleifolii, Verbesino auriculigerae-Siparunetum muricatae, Berberido beauverdianae-Myrcianthetum myrsinoidis, Aristeguietio discoloris-Kageneckietum lanceolatae). 


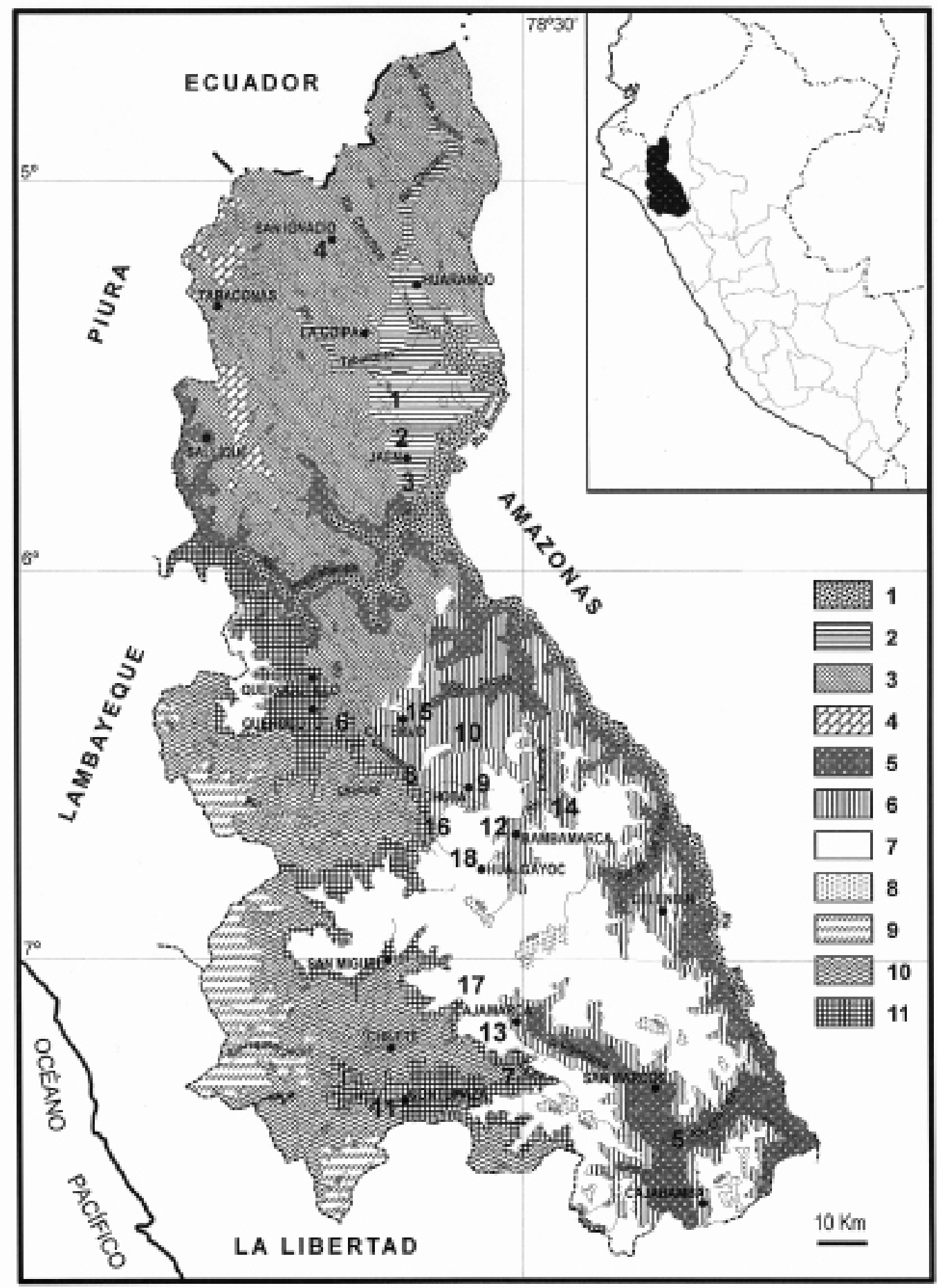


andina. Todo este contexto florístico es debido a la menor altitud alcanzada por los Andes en el territorio, propiciando mayor humedad, y a las cadenas montañosas transversales que originaron un elevado número de endemismos.

De todos los hábitats de Cajamarca, el que ha levantado más polémica ha sido el páramo (jalca) y su límite meridional, que ha sido considerado por la mayoría de autores como una unidad biogeográfica independiente del resto de la vegetación que lo rodea. En la tabla 9 indicamos el límite meridional del páramo según algunos autores. Cabrera \& Willink (1980), Hueck \& Seibert (1981), Rivas-Martínez \& Tovar (1982) y Brack Egg \& Mendiola Vargas (2004) indican que el páramo no alcanza territorio peruano. Sin embargo, Rivas-Martínez \& Tovar (1983), Rivas-Martínez et al. (2011), y Sánchez Vega \& Sánchez Rojas (2012) señalan a la depresión de Huancabamba $\left(5^{\circ} 45^{\prime}\right.$ a $6^{\circ} 19^{\prime}$ S $)$ como la barrera biogeográfica que separa el páramo, que queda en los Andes del norte, de la puna de los Andes centrales incluida la jalca. Incluso Ricardi et al. (1997) y Sánchez Vega \& Sánchez Rojas (2012) distinguen a la jalca como un pajonal más seco que el páramo, que se extiende desde la depresión de Huancabamba hasta el paralelo $8^{\circ} \mathrm{S}$, al inicio de la Cordillera Blanca.

Nuestra postura se une a la de Weberbauer (1936), Cleef (1978), Mostacero et al. (1996), Llatas et al. (1997), Luteyn (1999) y Weigend (2002, 2004), que llevan el páramo hasta las proximidades de los $8^{\circ} \mathrm{S}$; incluso este último autor lo une al resto de la vegetación describiendo la zona Amotape-Huancabamba.

Tal vez el modelo más apropiado para definir unidades biogeográficas es el de Rivas-Martínez et al. (2011), que recoge lo propuesto para el $\mathrm{N}$ del Perú por Llatas et al. (1997) y Galán de Mera et al. (2002), tomando como cliserie altitudinal el bosque seco occidental, bosque húmedo occidental, páramo (jalca), bosque húmedo oriental y bosque seco oriental en el río Marañón.
Sin embargo, respecto a la sectorización biogeográfica de Rivas-Martínez, llevamos la provincia Guayaquileña-Ecuatoriana hasta el paralelo $8^{\circ} \mathrm{S}$. Las razones son las siguientes: (1) La presencia de bosques secos, húmedos y de vegetación altoandina con una enorme cantidad de elementos comunes con Colombia y Ecuador (Galán de Mera et al., 2015), (2) la existencia de rodales de Puya fastuosa sobre suelos húmedos en el páramo $\mathrm{S}$ de Cajamarca, vicariantes de los de Puya santosii de Colombia (Rangel et al., 1997) y P. clava-herculis de Ecuador (Lauer et al., 2001), (3) la extensión de los humedales de Chusquea aristata Munro hasta Pasco y Cusco (11-13 ${ }^{\circ} \mathrm{S}$ en los Andes orientales)(Tropicos, 2015), y (4) la distribución de algunos grupos de especies dentro de los géneros Nasa Weigend, Passiflora L. y Ribes L. (Weigend, 2004).

No obstante, en el mapa de la figura 3, podemos separar dos sectores biogeográficos propiciados por la alineación montañosa NE-SO situada en las proximidades de Cutervo. El sector Loja-Cutervo se extiende al $\mathrm{N}$ de dicha alineación montañosa con los bosques húmedos de la clase Nectandro laurel-Licarietea canellae y el páramo de Oreobolo goeppingieriHypericetum laricifolii. Al S y O de Cutervo se inicia el sector Chota-Contumazá donde encontramos los bosques húmedos occidentales de la clase Pruno-Oreopanacetea floribundi, el páramo de Calamagrostio tarmensisHypericetum laricifolii, y un páramo superior de Agrostio tolucensis-Paspaletum bonplandiani, que incluye a Puya fastuosa. En la figura 4 mostramos un esquema de la vegetación de Cajamarca abarcando ambos sectores.

En consecuencia, la jerarquización biogeográfica del departamento de Cajamarca quedaría de la siguiente forma:

Reino Neotropical-Austroamericano

Subreino Neotropical

Región Neogranadina

Provincia Guayaquileña-Ecuatoriana

Sector Loja-Cutervo

Sector Chota-Contumazá 

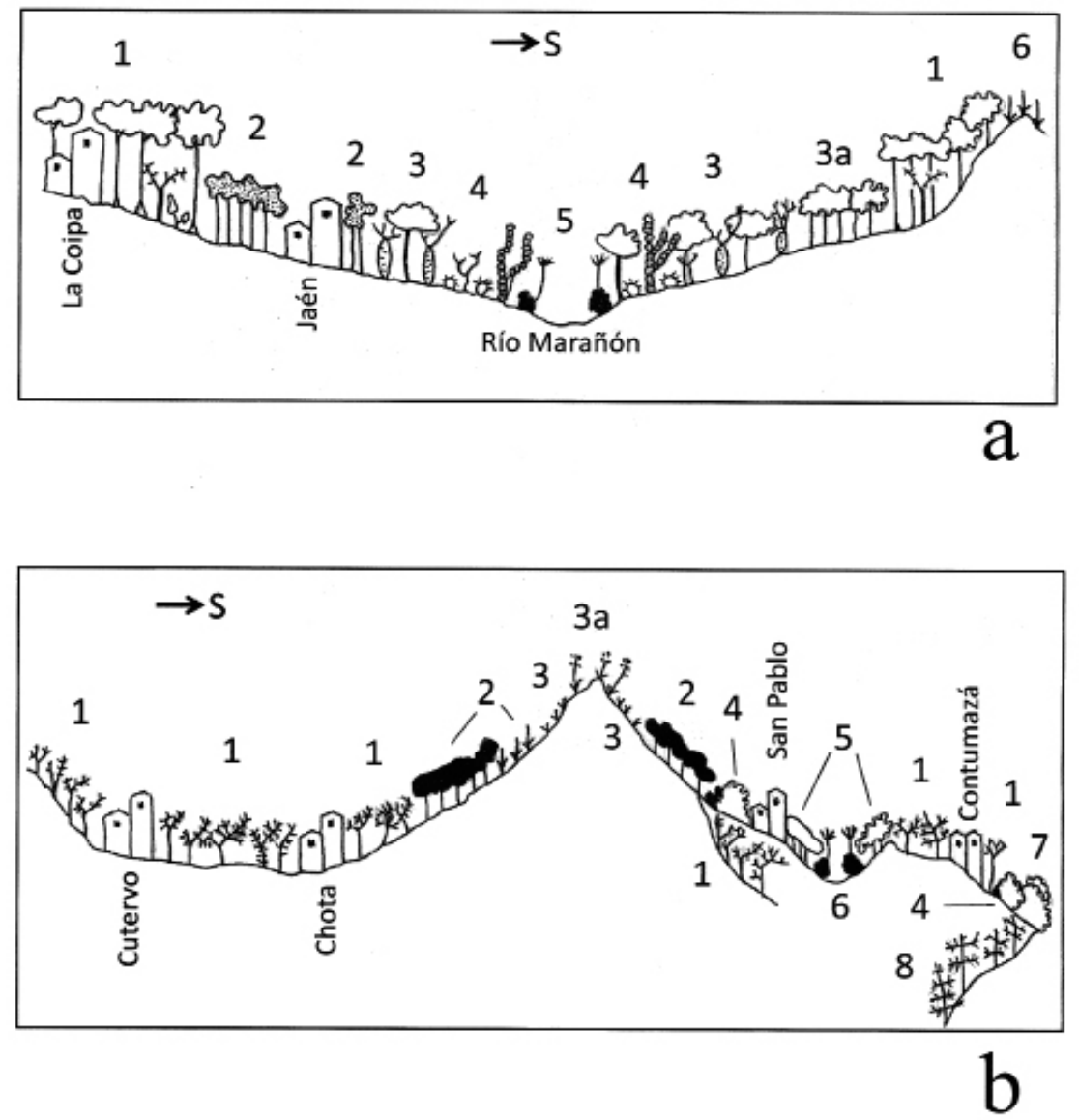

Figura 4. Esquema de la vegetación del departamento de Cajamarca entre La Coipa y Contumazá. a) Sector Loja-Cutervo: 1-Comunidad de Cecropia montana y Heliocarpus americanus, 2-Comunidad de Muntingia calabura y Hura crepitans, 3-Diplopterydo leiocarpae-Acacietum macracanthae, 3a-Diplopterydo leiocarpae-Acacietum macracanthae clusietosum pavonii, 4-Armatocereo balsasensis-Cercidietum praecocis, 5- Baccharito salicifoliae-Gynerietum sagittati, 6- Calamagrostio tarmensis-Hypericetum laricifolii; b) Sector Chota-Contumazá: 1- Valleo stipularis-Alnetum acuminatae, 2- Complejo de vegetación ligado a los bosques de Polylepis (Barnadesio dombeyanae-Polylepidetum racemosae, Baccharito latifoliaeMonactinetum flaverioidis, Calamagrostio tarmensis-Hypericetum laricifolii), 3-Agrostio tolucensisPaspaletum bonplandiani, 3a- Agrostio tolucensis-Paspaletum bonplandiani puyetosum fastuosae, 4- Aristeguietio discoloris-Kageneckietum lanceolatae, 5- Crotono ruiziani-Acacietum macracanthae, 6- Baccharito salicifoliae-Gynerietum sagittati, 7- Berberido beauverdianae-Myrcianthetum myrsinoidis, 8- Axinaeo nitidae-Podocarpetum oleifolii. Vegetation scheme of the Department of Cajamarca between La Coipa and Contumazá. a) Loja-Cutervo biogeographical sector: 1-Cecropia montana and Heliocarpus americanus plant community, 2- Muntingia calabura and Hura crepitans plant community, 3-Diplopterydo leiocarpae-Acacietum macracanthae, 3a-Diplopterydo leiocarpae-Acacietum macracanthae clusietosum pavonii, 4-Armatocereo balsasensis-Cercidietum praecocis, 5-Baccharito salicifoliae-Gynerietum sagittati, 6- Calamagrostio tarmensis-Hypericetum laricifolii; b) Chota-Contumazá biogeographical sector: 1Valleo stipularis-Alnetum acuminatae, 2-Vegetation complex linked to the Polylepis forests (Barnadesio dombeyanae-Polylepidetum racemosae, Baccharito latifoliae-Monactinetum flaverioidis, Calamagrostio tarmensis-Hypericetum laricifolii), 3-Agrostio tolucensis-Paspaletum bonplandiani, 3a-Agrostio tolucensisPaspaletum bonplandiani puyetosum fastuosae, 4- Aristeguietio discoloris-Kageneckietum lanceolatae, 5-Crotono ruiziani-Acacietum macracanthae, 6- Baccharito salicifoliae-Gynerietum sagittati, 7-Berberido beauverdianae-Myrcianthetum myrsinoidis, 8-Axinaeo nitidae-Podocarpetum oleifolii. 
Tabla 7

1-9: Calamagrostio tarmensis-Hypericetum laricifolii Galán, Sánchez, Montoya, Linares, Campos \& Vicente ass. nova; 10: cortaderietosum nitidae Galán, Sánchez, Montoya, Linares, Campos \& Vicente subass. nova

(Calamagrostietea vicunarum, Agrostio-Paspaletalia, Agrostio-Paspalion)

N. de inventario

Altitud (m)

Área $\left(\mathrm{m}^{2}\right)$

Pendiente (\%)

Orientación

Características de asociación

Hypericum laricifolium

Calamagrostis tarmensis

Calceolaria cajabambae

Paranephelius ferreyrii

Hieracium peruanum

Geranium peruvianum

Muhlenbergia caxamarcensis

Jungia stuebelii

Tridax peruviensis

Diferenciales de subasociación

Cortaderia nitida

Pitcairnia sp.

Características de alianza y orden Agrostis tolucensis

Stevia andina

Paspalum bonplandianum

Briza monandra

Festuca huamachucensis

Orthrosanthus chimboracensis

Lobelia tenera

Lupinus peruvianus

Arcythophyllum thymifolium

Bartsia adenophylla

Gaultheria glomerata

Gentianella bicolor

Valeriana pilosa

Hypericum aciculare

Alchemilla frigida

Tridax tambensis

Agrostis boliviana

Chrysactinium acaule

Antennaria linearifolia

Características de clase

Alchemilla propinqua

Werneria nubigena

Paranephelius ovatus

Pernettya prostrata

$\begin{array}{cccccccccccc}1 & 2 & 3 & 4 & 5 & 6 & 7 & 8 & 9 & 10 & \text { 宓 } & \text { 岕 } \\ 3566 & 3604 & 3578 & 3698 & 3555 & 3555 & 3708 & 3577 & 3699 & 3523 & - & - \\ 100 & 100 & 100 & 100 & 100 & 100 & 200 & 100 & 100 & 100 & - & - \\ . & \cdot & \cdot & . & . & . & 10 & 20 & 30 & 30 & - & - \\ . & . & . & . & . & . & \mathrm{NE} & \mathrm{N} & \mathrm{O} & \mathrm{S} & - & -\end{array}$

$\begin{array}{cccccccccccc}2 & 3 & 4 & . & 2 & 2 & 2 & 4 & 3 & 1 & * * & * * \\ 4 & 3 & . & 4 & . & 2 & . & 3 & 4 & . & * & * * \\ + & + & 1 & . & . & . & . & . & . & . & * & * \\ . & . & 1 & . & . & . & . & . & . & + & . & * \\ . & . & . & . & . & . & + & . & . & . & * & * * \\ . & . & . & . & . & . & . & . & + & . & * & * \\ 1 & . & . & . & . & . & . & . & . & . & . & * \\ . & . & 1 & . & . & . & . & . & . & . & . & * \\ . & . & . & . & . & . & 1 & . & . & . & . & .\end{array}$




\begin{tabular}{|c|c|c|c|c|c|c|c|c|c|c|c|c|}
\hline Baccharis caespitosa & . & . & + & 1 & . & . & 1 & 1 & . & & $* *$ & $*$ \\
\hline Castilleja nubigena & 1 & . & . & . & . & . & + & . & . & . & * & * \\
\hline Dioscorea ancashensis & . & . & . & + & . & . & . & + & 1 & . & . & $*$ \\
\hline Paranephelius uniflorus & . & . & . & . & 1 & . & 1 & . & 1 & . & . & $* *$ \\
\hline Eryngium humile & . & . & . & . & . & . & 1 & . & + & . & $* *$ & $* *$ \\
\hline Alchemilla procumbens & . & . & . & . & . & . & + & . & + & . & $*$ & . \\
\hline Hypericum silenoides & . & . & . & . & . & . & . & 1 & . & 1 & $*$ & ** \\
\hline Polgonatum oligodus & . & 3 & . & 2 & . & . & . & . & . & . & . & . \\
\hline Luzula racemosa & . & . & . & . & . & + & . & 1 & . & . & . & ** \\
\hline Trichophorum rigidum & . & . & . & . & . & 1 & . & . & . & . & $*$ & . \\
\hline Plantago sericea & . & . & . & . & . & . & . & 1 & . & . & $*$ & $*$ \\
\hline Aciachne pulvinata & . & . & . & . & . & . & . & 1 & . & . & $* *$ & $*$ \\
\hline Werneria villosa & . & . & . & . & . & . & . & . & + & . & $*$ & $*$ \\
\hline Bromus lanatus & . & . & 1 & . & . & . & . & . & . & . & . & . \\
\hline Phyllactis rigida & . & . & . & . & . & . & 1 & . & . & . & . & $*$ \\
\hline Muehlenbeckia volcanica & . & . & . & . & . & . & + & . & . & . & . & $* *$ \\
\hline Stipa macbridei & . & . & . & . & . & . & . & 1 & . & . & $*$ & . \\
\hline Novenia acaulis & . & . & . & . & . & . & . & 1 & . & . & . & . \\
\hline \multicolumn{13}{|l|}{ Compañeras } \\
\hline Alchemilla pinnata & + & 1 & 1 & 2 & . & . & . & . & . & . & $*$ & . \\
\hline Rumex angiocarpus & . & 1 & + & . & 1 & 1 & . & . & + & . & . & . \\
\hline Gnaphalium dombeyanum & 1 & 1 & . & . & + & . & . & . & + & . & . & $* *$ \\
\hline Nassella mucronata & . & 1 & 1 & . & 1 & 1 & . & . & . & . & . & $* *$ \\
\hline Hypochaeris chillensis & . & . & . & . & 1 & + & + & . & . & . & $*$ & $* *$ \\
\hline Briophyta & . & . & . & . & 1 & 2 & . & 3 & 2 & . & . & . \\
\hline Bidens triplinervia & 1 & 1 & . & . & . & . & + & . & . & . & . & $* *$ \\
\hline Achyrocline alata & . & 2 & 1 & . & . & . & . & . & . & . & $* *$ & $* *$ \\
\hline Alonsoa linearis & . & 1 & + & . & . & . & . & . & . & . & $*$ & . \\
\hline Alchemilla vulcanica & . & + & . & . & 2 & . & . & . & . & . & $*$ & . \\
\hline Monnina salicifolia & . & + & . & . & . & . & . & . & . & + & $*$ & $* *$ \\
\hline Ageratina exsertovenosa & . & . & . & . & . & + & . & . & . & 1 & $*$ & $* *$ \\
\hline Bartsia melampyroides & . & + & . & . & . & . & . & . & . & . & $*$ & $*$ \\
\hline Calamagrostis rigescens & . & . & . & 1 & . & . & . & . & . & . & $*$ & ** \\
\hline Polylepis racemosa & . & . & . & . & + & . & . & . & . & . & $*$ & $*$ \\
\hline Festuca glycerantha & . & . & . & . & 1 & . & . & . & . & . & $*$ & $*$ \\
\hline Anthoxanthum odoratum & . & . & . & . & . & 1 & . & 1 & . & . & . & . \\
\hline Gynoxys calyculisolvens & . & . & . & . & . & . & . & . & + & . & $* *$ & * \\
\hline Cerastium subspicatum & . & . & . & . & . & . & . & . & + & . & $* *$ & $*$ \\
\hline Valeriana interrupta & . & . & . & · & · & . & . & . & . & + & $*$ & $*$ \\
\hline Baccharis libertadensis & . & . & . & . & . & . & . & . & . & 1 & $*$ & $*$ \\
\hline Hesperomeles lanuginosa & . & . & . & . & . & . & . & . & . & 2 & $*$ & ** \\
\hline
\end{tabular}

Otras compañeras. En 1: Rumex acetosella 1. En 2: Trifolium amabile 1. En 3: Gnaphalium americanum +, Vicia graminea + , Hyptis obtusata + . En 4: Austrocylindropuntia floccosa + . En 5: Bidens andicola + , Stachys aperta 1. En 9: Salvia sp. + . En 10: Baccharis nitida, +.Disterigma empetrifolium, + .

Localidades. 1-3- Cajamarca, Agua Tapada (1- 17M 0769541-9204164, 2- 17M 0769493-9204242, 3- 17M 07694629204226), 4- Cajamarca, Secsemayo (17M 0765550-9206922), 5-6- Cajamarca, Granja Porcón (17M 07622019218888), 7- Cajamarca, Porcón Alto, La Fila (17M 0756170-9216828), 8-9-Cajamarca, Contumazá, Camino a Pozo Kuan (8- 17M 0752140-9182366, 9- 17M 0752713-9182508), 10- Cajamarca, Porcón Alto, La Fila (17M 07574719218214). Géneros $(*)$ y especies (**) también presentes en Sabogal (2014) y Peyre (2015). Genera (*) and species (**) also present in Sabogal (2014) and Peyre (2015). 
Tabla 8

1-2: Agrostio tolucensis-Paspaletum bonplandiani Galán, Sánchez, Montoya, Linares, Campos \& Vicente ass. nova; 3-4: puyetosum fastuosae Galán, Sánchez, Montoya, Linares,

Campos \& Vicente subass. nova; 5-6: calamagrostietosum curvulae Galán, Sánchez,

Montoya, Linares, Campos \& Vicente subass. nova

(Calamagriostietea vicunarum, Agrostio-Paspaletalia, Agrostio-Paspalion)

N. de inventario

Altitud (m)

Área $\left(\mathrm{m}^{2}\right)$

Pendiente \%

Orientación

Carácterísticas de asociación

Paspalum bonplandianum

Agrostis tolucensis

Werneria stuebelii

Ascidiogyne sanchezvegae

Diferenciales de subasociaciones

Calamagrostis rigescens

Puya fastuosa

Lycopodium clavatum

Loricaria ferruginea

Calamagrostis curvula

Nassella depauperata

Mniodes pulvinata

Alchemilla frigida

Características de alianza y orden

Festuca huamachucensis

Oritrophium peruvianum

Arcytophyllum filiforme

Bartsia adenophylla

Aphanactis villosa

Antennaria linearifolia

Calamagrostis tarmensis

Geranium multipartitum

Belloa turneri

Lupinus peruvianus

Poa pauciflora

Características de clase

Trichophorum rigidum

Paranephelius uniflorus

Eryngium humile

Werneria villosa

Luzula racemosa

Plantago sericea

Oreithales integrifolia

Baccharis caespitosa

Senecio tephrosioides

$\begin{array}{cccccc}1 & 2 & 3 & 4 & 5 & 6 \\ 3881 & 3850 & 3891 & 3858 & 3995 & 3982 \\ 100 & 100 & 100 & 200 & 100 & 100 \\ 20 & 20 & 30 & 20 & 5 & 5 \\ \mathrm{~N} & \mathrm{~N} & \mathrm{~N} & \mathrm{~N} & \mathrm{~S} & \mathrm{~S} \\ & & & & & \\ 5 & 5 & 5 & 4 & 2 & 3 \\ 4 & 3 & 3 & 2 & 1 & 1 \\ 1 & 1 & 2 & 2 & 1 & 1 \\ 2 & 1 & . & . & . & .\end{array}$




\section{Hypericum silenoides}

Ranunculus praemorsus

\section{Caracteristicas de Anthochloo lepidulae-Dielsiochloetea floribundae}

Werneria apiculata

Belloa piptolepis

Novenia acaulis

Azorella biloba

\section{Caracteristicas de Plantagini-Distichietea y Plantaginetea australis}

Sisyrinchium pusillum

Nierembergia repens

Gentiana sedifolia

Phyllactis rigida

Otras compañeras. En 1: Muhlenbergia peruviana + , Castilleja pumila + , Vulpia dertonensis + , Hypochaeris taraxacoides 1. En 2: Hypochaeris taraxacoides + , Briophyta 2. En 3: Hypochaeris taraxacoides 1, Briophyta 2. En 5: Nassella mucronata +, Calceolaria micans +, Gamochaeta purpurea + .

Localidades. 1-4- Cajamarca, Coimolache (1- 17M 0761892-9236996, 2- 17M 0792800-9264547; 3 17M 0761925-9236968, 4- 17M 0764616-9232922). 5-6- Cajamarca, Contumazá, Pozo Kuan (5-17M 0755611-9182856, 6- 17M 0755505-9182804).

Tabla 9

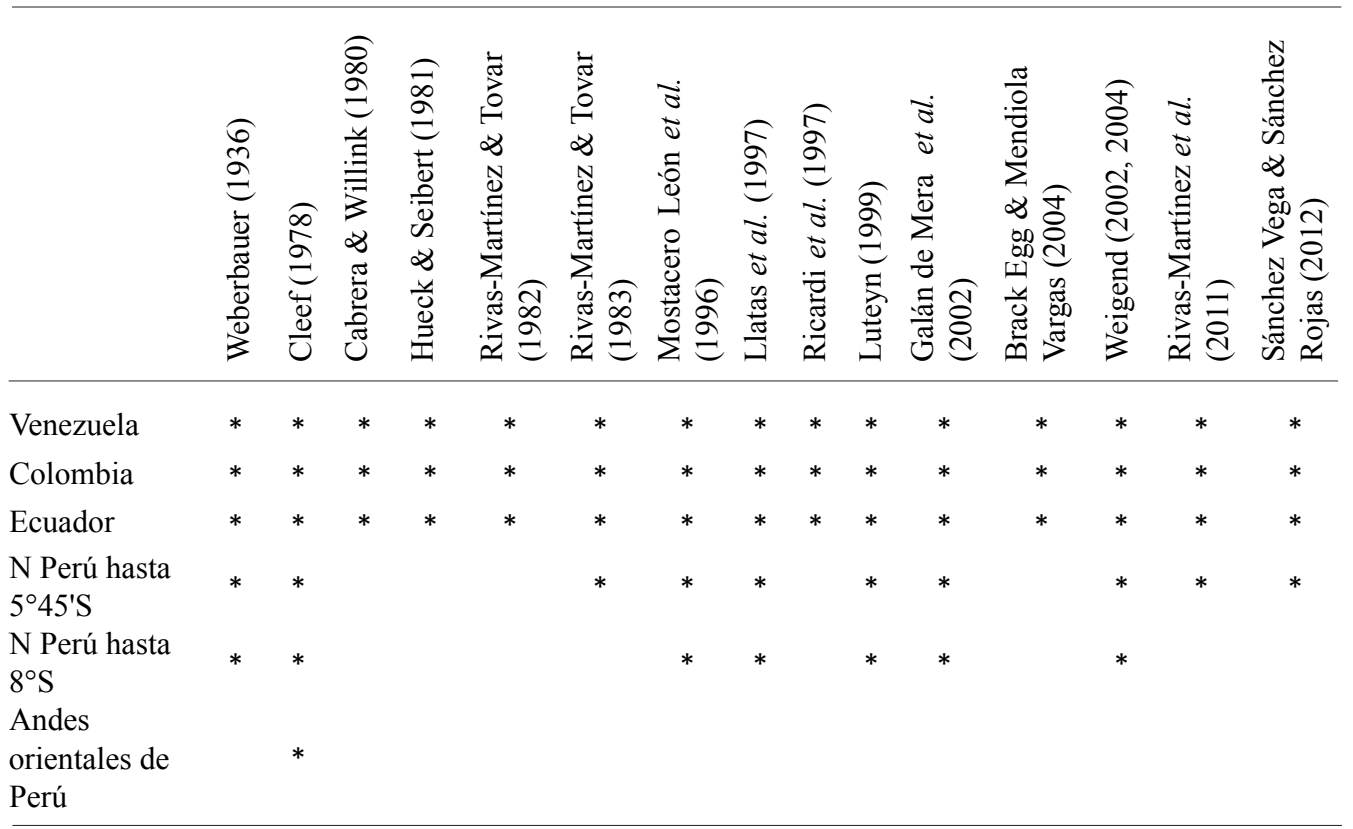

Tabla 9. Límite meridional del páramo según diversos autores, teniendo en cuenta la depresión de Huancabamba $\left(5^{\circ} 45^{\prime} \mathrm{S}\right), \operatorname{los} 8^{\circ} \mathrm{S}$, y su presencia en los Andes orientales peruanos. Southern limit of the paramo according to different authors, taking account of the Huancabamba Depression ( $\left.5^{\circ} 45^{\prime} S\right)$, the latitude $8^{\circ} S$, and its presence in the oriental Peruvian Andes. 


\section{CONCLUSIONES}

Como conclusión, damos a conocer el esquema sintaxonómico de las unidades de vegetación del departamento de Cajamarca ordenadas por formaciones vegetales, junto a una pequeña diagnosis de las comunidades, asociaciones y subasociaciones.

\section{ESQUEMA SINTAXONÓMICO}

\section{Bosques secos y comunidades de Cactáceas} ACACIO MACRACANTHAE-PROSOPIDETEA PALLIDAE Galán de Mera 1999

+ Cryptocarpo pyriformis-Prosopidetalia pallidae Galán de Mera \& Cáceres in Galán de Mera, Rosa \& Cáceres 2002

* Bursero graveolentis-Prosopidion pallidae Galán de Mera \& Cáceres in Galán de Mera, Rosa \& Cáceres 2002

1. Loxopterygio huasanginis-Neoraimondietum arequipensis Galán de Mera, Sánchez Vega, Linares, Campos \& Vicente 2015

[Asociación occidental de Cactáceas infratropical árido-semiárida]

2. Crotono ruiziani-Acacietum macracanthae Galán de Mera, Sánchez Vega, Linares, Campos \& Vicente 2015

[Bosques occidentales secos espinosos termotropicales]

* Baccharito oblongifoliae-Jacarandion acutifoliae Galán de Mera, Sánchez Vega, Linares, Campos \& Vicente 2015

3. Armatocereo balsasensis-Cercidietum praecocis Galán de Mera, Sánchez Vega, Linares, Campos \& Vicente 2015

[Asociación oriental de Cactáceas infratropical semiárida]

4. Diplopterydo leiocarpae-Acacietum macracanthae Galán de Mera, Sánchez Vega, Linares, Campos \& Vicente 2015

[Bosques orientales secos espinosos termotropicales] acacietosum macracanthae

flourensietosum cajabambensis Galán de Mera, Sánchez Vega, Linares, Campos \& Vicente 2015 [subasociación seca del sector Chota-Contumazá] clusietosum pavonii Galán de Mera, Sánchez Vega, Linares, Campos \& Vicente 2015 [subasociación subhúmeda del valle del Marañón]

II. Pluvisilvas andinas

NECTANDRO LAEVIS-LICARIETEA CANELLAE Izco 2013

+ Nectandro laevis-Clusietalia emarginatae Izco 2013

* Tibouchino lepidotae-Vismion tomentosae Izco 2013

5. Comunidad de Cecropia montana y Heliocarpus americanus

[Bosques termotropicales subhúmedo-húmedos del sector Loja-Cutervo]

6. Comunidad de Muntingia calabura y Hura crepitans

[Bosques infratropicales seco-subhúmedos del sector Loja-Cutervo]

III. Bosques lauroides y esclerófilos occidentales PRUNO RIGIDAE-OREOPANACETEA FLORIBUNDI Galán de Mera 2005

+ Cestro auriculati-Prunetalia rigidae Galán de Mera \& Rosa in Galán de Mera, Rosa \& Cáceres 2002

* Monnino pilosae-Myrcianthion myrsinioidis Galán de Mera, Sánchez Vega, Montoya, Linares, Campos \& Vicente all. nova

7. Axinaeo nitidae-Podocarpetum oleifolii Galán de Mera, Sánchez Vega, Montoya, Linares, Campos \& Vicente ass. nova

[Bosque lauroide mesotropical húmedohiperhúmedo]

8. Verbesino auriculigerae-Siparunetum muricatae Galán de Mera, Sánchez Vega, Montoya, Linares, Campos \& Vicente ass. nova

[Bosque lauroide mesotropical húmedo-hiperhúmedo derivado de la acción antrópica]

9. Berberido beauverdianae-Myrcianthetum myrsinoidis Galán de Mera, Sánchez Vega, Montoya, Linares, Campos \& Vicente ass. nova

[Bosque esclerófilo mesotropical inferior secosubhúmedo]

10. Aristeguietio discoloris-Kageneckietum lanceolatae Galán de Mera, Sánchez Vega, Montoya, Linares, Campos \& Vicente ass. nova

[Bosque esclerófilo mesotropical superior secosubhúmedo]

IV. Alisedas andinas

ALNETEA ACUMINATAE Galán de Mera 2005

+ Alnetalia acuminatae Galán de Mera \& Rosa in Galán de Mera, Rosa \& Cáceres 2002

* Myrico pubescentis-Alnion acuminatae Galán de Mera \& Rosa in Galán de Mera, Rosa \& Cáceres 
2002

11. Valleo stipularis-Alnetum acuminatae Galán de Mera \& Rosa in Galán de Mera, Rosa \& Cáceres 2002

[Alisedas supra-mesotropicales seco-subhúmedas de suelos con humedad edáfica]

V. Bosques altoandinos

POLYLEPIDETEA TARAPACANO-BESSERI

Rivas-Martínez \& Navarro in Navarro \& Maldonado 2002

+ Polylepidetalia racemosae Galán de Mera \& Cáceres in Galán de Mera, Rosa \& Cáceres 2002

* Polylepidion incano-pautae Galán de Mera 2005

12. Barnadesio dombeyanae-Polylepidetum racemosae Galán de Mera, Sánchez Vega \& Linares 2013

[Queñuales supratropicales subhúmedo-húmedos del N del Perú]

VI. Arbustedas

CLEMATIDOPERUVIANAE-BACCHARITETEA

LATIFOLIAE Galán de Mera, Sánchez Vega, Montoya, Linares, Campos \& Vicente cl. nova

+ Mutisio acuminatae-Baccharitetalia latifoliae Galán de Mera \& Cáceres in Galán de Mera, Rosa \& Cáceres 2002

* Otholobio munyensis-Rubion robusti Galán de Mera, Sánchez Vega, Montoya, Linares, Campos \& Vicente all. nova

13. Baccharito latifoliae-Monactinetum flaverioidis Galán de Mera, Sánchez Vega, Montoya, Linares, Campos \& Vicente ass. nova

[Arbustedas heliófilas supra-mesotropicales subhúmedas-secas]

14. Monactino flaverioidis-Colignonietum parviflorae Galán de Mera, Sánchez Vega, Montoya, Linares, Campos \& Vicente ass. nova prov.

[Arbustedas mesotropicales húmedas]

VII. Pajonales del páramo (jalca)

CALAMAGROSTIETEA VICUNARUM

Rivas-Martínez \& Tovar 1982

+ Agrostio tolucensis-Paspaletalia bonplandiani

Galán de Mera, Sánchez Vega, Montoya, Linares, Campos \& Vicente ordo novo

* Agrostio tolucensis-Paspalion bonplandiani Galán de Mera, Sánchez Vega, Montoya, Linares, Campos \& Vicente all. nova

15. Oreobolo goeppingieri-Hypericetum laricifolii Sabogal 2014

[Pajonales supratropicales húmedos del sector Loja-Cutervo]
16. Calamagrostio tarmensis-Hypericetum laricifolii Galán de Mera, Sánchez Vega, Montoya, Linares, Campos \& Vicente ass. nova

[Pajonales supratropicales húmedos del sector ChotaContumazá]

hypericetosum laricifolii

cortaderietosum nitidae Galán de Mera, Sánchez Vega, Montoya, Linares, Campos \& Vicente subass. nova [Subasociación de áreas rocosas]

17. Agrostio tolucensis-Paspaletum bonplandiani Galán de Mera, Sánchez Vega, Montoya, Linares, Campos \& Vicente ass. nova

[Pajonales orotropicales húmedo-hiperhúmedos] paspaletosum bonplandiani

puyetosum fastuosae Galán de Mera, Sánchez Vega, Montoya, Linares, Campos \& Vicente subass. nova [Áreas con humedad edáfica]

calamagrostietosum curvulae Galán de Mera, Sánchez Vega, Montoya, Linares, Campos \& Vicente subass. nova [Subasociación de transición a la puna centroperuana]

VIII. Vegetación de riberas

TESSARIO INTEGRIFOLIAE-BACCHARITETEA

SALICIFOLIAE Rivas-Martínez \& Navarro in

Navarro \& Maldonado 2002

+ Plucheo absinthioidis-Baccharitetalia salicifoliae

Rivas-Martínez \& Navarro in Navarro \& Maldonado 2002

* Pityrogrammo trifoliatae-Baccharition salicifoliae Galán de Mera, Baldeón, Beltrán, Benavente \& Gómez 2004

18. Baccharito salicifoliae-Gynerietum sagittati Galán de Mera, Baldeón, Beltrán, Benavente \& Gómez 2004

[Comunidades andinas de grandes helófitos de cauces pedregosos y aguas corrientes]

AGRADECIMIENTOS. Dedicamos este trabajo a la memoria de nuestro amigo y maestro Prof. Dr. Isidoro Sánchez Vega (Don Ishico), que durante nuestros viajes por Cajamarca nos transmitió sus profundos conocimientos sobre flora, vegetación y antropología, y nos alentó siempre con su sencillez hasta sus últimos momentos. Este trabajo ha sido realizado gracias a los fondos del proyecto "Mapa de vegetación de Cajamarca. Potencialidad de la vegetación para el uso de plantas medicinales" de la Universidad Privada Antonio Guillermo Urrelo-UPAGU (Cajamarca, Perú), dentro del convenio firmado con la Universidad San Pablo-CEU (Madrid, España). Gracias al personal 
de los herbarios consultados que pusieron a nuestra disposición el material necesario para la realización de este estudio. El profesor Iván Torres, de la UPAGU de Cajamarca, nos ayudó con todo tipo de recursos durante los trabajos de campo y estadía en Cajamarca. Los profesores Betty Millán, Haydee Montoya y José Gómez, de la Universidad Nacional Mayor de San Marcos (Lima, Perú), una vez más, nos brindaron un excelente apoyo durante nuestros estudios en Lima. Gracias a Julio Pinto, que por entender el trabajo con plantas, trajo los "zoquetes" y manejó con cautela sobre barro y nieve. Nuestro agradecimiento a Missouri Botanical Garden que facilitó el acceso a los transectos de A.H. Gentry, y a los revisores anónimos que mejoraron el manuscrito.

\section{BIBLIOGRAFÍA}

BRACK EGG, A. \& C. MENDIOLA VARGAS -2004- Ecología del Perú. Bruño. Lima.

BRAKO, L. \& J.L. ZARUCCHI -1993- Catálogo de las Angiospermas y Gimnospermas del Perú. Missouri Botanical Garden. St. Louis.

BRAUN-BLANQUET, J. -1979- Fitosociología. H. Blume. Madrid.

BUSSMANN, R.W. -2003- The vegetation of Reserva Biológica San Francisco, Zamora-Chinchipe, Southern Ecuador- a phytosociological synthesis. Lyonia 3(2): 145-254.

CABRERA, A.L. \& A. WILLINK -1980Biogeografía de América Latina. Organización de los Estados Americanos. Washington, DC.

CLEEF, A.M. -1978- Characteristics of neotropical páramo vegetation and its sub-Antarctic relations. In Troll, C. \& W. Lauer (eds.) Geoecological relations between the southern temperate zone and the tropical mountains: 365-390. Erdwissenschaftliche Forschung 11. Franz Steiner. Wiesbaden.

CUATRECASAS, J. -2003- Observaciones geobotánicas en el Nevado del Tolima, Cordillera Central, en 1932. In Van der Hammen, T. \& A.G. Dos Santos (eds.) La Cordillera Central Colombiana. Transecto Parque Los Nevados V: 241-309. Estudios de Ecosistemas Tropandinos. J. Cramer. Berlin-Stuttgart.

DENGLER, J., M. CHYTRÝ \& J. EWALD -2008Phytosociology. In Jørgensen, S.E. \& B.D. Fath (eds.) Encyclopedia of Ecology: 2767-2779. Elsevier. Oxford.

GALÁN DE MERA, A. -2005-Clasificación fitosociológica de la vegetación de la región del Caribe y América del Sur. Arnaldoa 12(1-2): 86-111.

GALÁN DE MERA, A., S. BALDEÓN, H. BELTRÁN, M. BENAVENTE \& J. GÓMEZ -2004- Datos sobre la vegetación del centro del Perú. Acta Bot. Malacitana 29: 89-115.

GALÁN DE MERA, A. \& E. LINARES PEREA -2012- La vegetación de la región Arequipa (Perú). Universidad Nacional de San Agustín. Arequipa.

GALÁN DE MERA, A., E. LINARES PEREA, J. CAMPOS DE LA CRUZ, C. TRUJILLO VERA, F. VILLASANTE BENAVIDES \& J.A. VICENTE ORELLANA -2011- Novedades sobre la vegetación del Departamento de Arequipa (Perú). Arnaldoa 18(2): 125-144.

GALÁN DE MERA, A., E. MÉNDEZ, E. LINARES PEREA, J. CAMPOS DE LA CRUZ \& J.A. VICENTE ORELLANA -2014- Las comunidades vegetales relacionadas con los procesos criogénicos en los Andes peruanos. Phytocoenologia 44(1-2): 121-161.

GALÁNDE MERA,A., M.V. ROSA\& C. CÁCERES -2002- Una aproximación sintaxonómica sobre la vegetación del Perú. Clases, órdenes y alianzas. Acta Bot. Malacitana 27: 75-103.

GALÁN DE MERA, A., I. SÁNCHEZ VEGA \& E. LINARES PEREA -2013- Pisos bioclimáticos y vegetación en la región de Cajamarca (Perú). Perspectiva 14(16): 119-129.

GALÁN DE MERA, A., I. SÁNCHEZ VEGA, E.LINARES PEREA, J. CAMPOS, J. MONTOYA \& J.A. VICENTE ORELLANA -2015-A phytosociological analysis and sinopsis of the dry woodlands and succulent vegetation of the Peruvian Andes. Anais Acad. Brasil. Ci. 87 (en prensa).

GALÁN DE MERA, A. \& J.A. VICENTE ORELLANA -2007- Cronosequences of vegetation- a bioclimatic theory for interpreting the patterns of relic vegetation types. Phytocoenologia 37: 471-494.

GALÁN DE MERA, A., J.A. VICENTE ORELLANA, E. LINARES PEREA, J. CAMPOS DE LA CRUZ, C. TRUJILLO VERA \& F. VILLASANTE BENAVIDES -2012Patrones de distribución de las comunidades de Cactáceas en las vertientes occidentales de los Andes peruanos. Caldasia 34: 257-275.

GÉHU, J.M. \& S. RIVAS-MARTÍNEZ -1981- 
Notions fondamentales de Phytosociologie. In Dierschke, H. (ed.) Syntaxonomie: 5-33. J. Cramer. Vaduz.

GENTRY, A.H. -1993- A Field Guide to the Families and Genera of Woody Plants of Northwest South America (Colombia, Ecuador, Peru) with Supplementary Notes on Herbaceous Taxa. Conservation International. Washington D.C.

GENTRY, A.H. -1995-2015- The Alwyn H. Gentry Forest Transect Data Set (fecha de consulta 18/06/15) Missouri Botanical Garden. St. Louis. http://www.mobot.org/mobot/research/gentry/ welcome.shtml\#bignon.

GUTTE, P. -1985- Beitrag zur Kenntnis zentralperuanischer Pflanzengesellschaften IV. Die grasreiche Vegetation der alpine Stufe. Wiss. Z. Karl-Marx-Univ. Leipzig, Math.-Naturwiss. R. 34(4): 357-401.

HENSOLD, N. -1999- Las angiospermas del Departamento de Cajamarca, Perú. Arnaldoa 6: 141-184.

HUECK, K. \& P. SEIBERT -1981- Vegetationskarte von Südamerika. Gustav Fischer. Stuttgart-New York.

INSTITUTO DE GEOLOGÍA Y MINERÍA -1975Mapa Geológico del Perú. Escala 1: 1.000.000. Ministerio de Energía y Minas. Lima.

IZCO, J. -2013- The Biological Reserve of San Francisco (Ecuador): revision of the syntaxonomy and nomenclature of the vegetation. Lazaroa 34 : 257-266.

JØRGENSEN, P.M. \& S. LEÓN-YÁÑEZ -1999Catalogue of the Vascular Plants of Ecuador. Missouri Botanical Garden. St. Louis.

JØRGENSEN, P.M., C. ULLOA, J.E. MADSEN \& R. VALENCIA -1995- A floristic analysis of the high Andes of Ecuador. In Churchill, S.P., H. Balslev, E. Forero \& J.L. Luteyn (eds.) Biodiversity and conservation of neotropical montane forests: 221-238. New York Botanical Garden. Bronx.

JUÁREZ, A.M., J.E. AYASTA, R.P. AGUIRRE \& E.F. RODRÍGUEZ -2005- La Oscurana (Cajamarca), un bosque relicto más para conservar en las vertientes occidentales andinas del norte del Perú. Rev. peru. biol. 12(2): 289-298.

LAUER, W., M.D. RAFIQPOOR \& I. THEISEN -2001-Physiographie, Vegetation und Syntaxonomie der Flora des Páramo de Papallacta (Ostkordillere Ecuador). Franz
Steiner. Stuttgart.

LISSON, G. -1980- Cómo se generó el suelo peruano. Contribución a la paleogeografía del Perú y las causas del relieve actual. In Samame Boggio, M. (ed.) El Perú Minero, III. Geología: 61-82. Ingemmet. Lima.

LLATAS QUIRÓS, S., A. GALÁN DE MERA \& J.A. VICENTE ORELLANA -1997- Proyecto de una flora del Departamento de Lambayeque (Perú). Anales Jard. Bot. Madrid 55(1): 176-181.

LLATAS QUIRÓS, S. \& M. LÓPEZ-MESONES -2005- Bosques montanos-relictos en Kañaris (Lambayeque, Perú). Rev. peru. biol. 12(2): 299-308.

LUTEYN, J.L. -1999- Páramos. A checklist of plant diversity, geographical distribution, and botanical literature. New York Botanical Garden. Bronx.

MARCELO PEÑA, J.L. \& M.A. LATORRE CUADROS -2010- Flora y vegetación del páramo adyacente a las Lagunas Arrebiatadas en el Santuario Nacional Tabaconas Namballe (Tabaconas, San Ignacio, Cajamarca)(fecha de consulta 30/06/15). Mecanismo de Información de los Páramos. Quito. http://www.paramo.org/ node/1544.

MARCELO PEÑA, J.L., I. SÁNCHEZ VEGA \& J.F. MILLÁN TAPIA -2006- Estado actual de la diversidad florística del Páramo. Sectores: El Espino y Palambe, Sallique, Jaén. Cajamarca. Perú. Ecol. Apl. 5 (1,2): 1-8.

MEZ, C. -1907- Additamenta monographica 1906. I. Bromeliaceae. Repert. Spec. Nov. Regni Veg. 3: 4-15.

MOSTACERO LEÓN, J., F. MEJÍA COICO \& F. PELÁEZ PELÁEZ -1996- Fitogeografía del Norte del Perú. Concytec. Lima.

PEYRE, G. -2015- Plant diversity and vegetation of the Andean Páramo. Tesis doctoral inédita. Universidad de Barcelona-Aarhus University.

PHILLIPS, O. \& J.S. MILLER -2002- Global Patterns of Plant Diversity: Alwyn H. Gentry's Forest Transect Data Set. Missouri Botanical Garden. St. Louis.

RANGEL, J.O. -2015- La biodiversidad de Colombia: significado y distribución regional. Rev. Acad. Colomb. Cienc. Ex. Fis. Nat. 39(151): 176-200.

RANGEL, J.O., P.D. LOWY \& M. AGUILAR -1997- Tipos de vegetación en Colombia. Colombia Diversidad Biótica II. Universidad 
Nacional de Colombia. Bogotá.

RAYNEL, C., R.T. PENNINGTON \& T. SÄRKINEN -2013- Cómo se formó la diversidad ecológica del Perú. Fundación Desarrollo Agrario-Royal Botanic Garden. Lima-Edinburgh.

RICARDI, M.H., J. GAVIRIA \& J. ESTRADA -1997- La Flora del Superpáramo venezolano y sus relaciones fitogeográficas a los largo de Los Andes. Plantula 1(3): 171-187.

RIVAS-MARTÍNEZ, S., G. NAVARRO, A. PENAS \& M. COSTA -2011- Biogeographic Map of South America. A preliminary survey. Int. J. Geobot. Res. 1: 21-40 + Map.

RIVAS-MARTÍNEZ, S. \& S. RIVAS-SÁENZ -1996-2015- Worldwide Bioclimatic Classification System (fecha de consulta 06/07/15). Phytosociological Research Center. Madrid. http://www.globalbioclimatics.org.

RIVAS-MARTÍNEZ, S. \& O. TOVAR -1982Vegetatio Andinae, I. Datos sobre las comunidades vegetales altoandinas de los Andes Centrales del Perú. Lazaroa 4: 167-187.

RIVAS-MARTÍNEZ, S. \& O. TOVAR -1983Síntesis biogeográfica de los Andes. Collect. Bot. (Barcelona) 14: 515-521.

SABOGAL, A. -2014- Ecosistemas del páramo peruano. Concytec. Lima.

SAGÁSTEGUI ALVA, A. -1989-Vegetación y flora de la provincia de Contumazá. Concytec. Trujillo.

SAGÁSTEGUI ALVA, A., S. LEIVA GONZÁLEZ, P. LEZAMAASENCIO, N. HENSOLD \& M.O. DILLON -1995- Inventario preliminar de la flora del Bosque Cachil. Arnaldoa 3(2): 19-34.

SAGÁSTEGUI ALVA, A., M.O. DILLON, I. SÁNCHEZ VEGA, S. LEIVA GONZÁLEZ \& P. LEZAMA ASENCIO -1999- Diversidad Florística del Norte del Perú, I. WWFPerú-Universidad Privada Antenor Orrego. Lima-Trujillo.

SAGÁSTEGUI ALVA, A., I. SÁNCHEZ VEGA, M. ZAPATA CRUZ \& M.O. DILLON -2003Diversidad Florística del Norte del Perú, II. Bosques montanos. Universidad Privada Antenor Orrego. Trujillo.

SÁNCHEZ VEGA, I. \& A. SÁNCHEZ ROJAS -2012- La Diversidad Biológica en Cajamarca. Visión étnico-cultural y potencialidades. Gobierno Regional. Cajamarca.

SCHJELLERUP, I., M.K. SØRENSEN, C. ESPINOZA, V. QUIPUSCOA \& V. PEÑA
-2003- Los valles olvidados. Pasado y presente en la utilización de recursos en la ceja de selva, Perú. The National Museum of Denmark. Copenhagen.

SEIBERT, P. \& X. MENHOFER -1991-Die Vegetation des Wohngebietes der Kallawaya und des Hochlandes von Ulla-Ulla in den bolivianischen Anden. Phytocoenologia 20(2): 145-276.

THE PLANT LIST -2015- The Plant List. A working list of all plant species (fecha de consulta 15/08/2015). Kew Royal Botanic Gardens, Missouri Botanical Garden, The New York Botanical Garden. http://www.theplantlist.org.

TROPICOS -2015- Tropicos.org. Missouri Botanical Garden. St. Louis.

ULLOA ULLOA, C. \& P.M. JØRGENSEN -2015Árboles y arbustos de los Andes del Ecuador. Missouri Botanical Garden (www.eFloras.org).

VAN DER HAMMEN, T. -2003- Ecosistemas zonales en los flancos oeste y este de la Cordillera Central Colombiana. In Van der Hammen, T. \& A.G. Dos Santos (eds.) La Cordillera Central Colombiana. Transecto Parque Los Nevados V: 503-545. Estudios de Ecosistemas Tropandinos. J. Cramer. Berlin-Stuttgart.

VICUÑA-MIÑANO, E.E. -2005- Las Podocarpáceas de los bosques montanos del noroccidente peruano. Rev. peru. biol. 12(2): 283-288.

WEBERBAUER, A. -1936- Phytogeography of the Peruvian Andes. In MacBride, J.F. (ed.) Flora of Peru 13(1): 13-81. Field Museum of Natural History. Chicago.

WEBER, H.E., J. MORAVEC \& J.P. THEURILLAT -2000- International Code of Phytosociological Nomenclature. 3rd edition. J. Veg. Sci. 11: 739-768.

WEIGEND, M. -2002- Observations on the Biogeography of the Amotape-Huancabamba Zone in Northern Peru. Bot. Rev. 68(1): 38-54.

WEIGEND, M -2004- Additional observations on the biogeography of the Amotape-Huancabamba zone in Northern Peru: Defining the South-Eastern limits. Rev. peru. biol. 11(2): 127-134.

WEIGEND, M., E.F. RODRÍGUEZ \& C. ARANA -2005- The relict forests of Northwest Peru and Southwest Ecuador. Rev. peru. biol. 12(2): 185-194.

WEIGEND, M. \& E.F. RODRÍGUEZ -2005Conservación de los bosques relictos del $\mathrm{NO}$ de Perú. Rev. peru. biol. 12(2): 335-336. 